\title{
LOCAL COEFFICIENTS AS ARTIN FACTORS FOR REAL GROUPS
}

\author{
FREYDOON SHAHIDI
}

\section{To Guity}

Introduction. The purpose of this paper is to prove the equality of certain local coefficients of arithmetic significance which were attached to representations of quasi-split real reductive algebraic groups in [27] with their corresponding Artin factors attached by local class field theory [21]. As a consequence, we establish an identity satisfied by certain normalized intertwining operators. It seems to be useful in applications of the trace formula [1,29].

More precisely, let $G$ be the group of real points of a quasi-split reductive algebraic group over $\mathbf{R}$. Let $\Delta$ be the set of simple roots defined by a fixed minimal parabolic subgroup $P_{0}=M_{0} A_{0} U$ of $G$. Fix $\theta \subset \Delta$, and let $P=P_{\theta}$ be the corresponding standard parabolic subgroup of $G$ and write $P=M A N$ for its Langlands decomposition. Fix a nondegenerate character $\chi$ of $U$ and let $(\sigma, H(\sigma))$ be an irreducible admissible $\chi$-generic Banach (in particular $\chi$-generic unitary) representation of $M$ (cf. Section 1). Given $\nu \in \mathfrak{a}_{\mathbf{C}}^{*}$, the complex dual of the Lie algebra of $A$, let $I(\nu, \sigma, \theta)$ be the continuously (quasi-unitarily, if $\sigma$ is unitary) induced representation $\operatorname{Ind}_{P \uparrow G} \sigma \otimes e^{\nu}$, and let $V(\nu, \sigma, \theta)$ be its space (Section 0). Then $V(\nu, \sigma, \theta)_{\infty}=V\left(\nu, \sigma_{\infty}, \theta\right)$.

Now, let $W$ be the Weyl group of $A_{0}$ in $G$. Choose $\tilde{w} \in W$ such that $\tilde{w}(\theta) \subset \Delta$. Let $N^{-}$be the unipotent group opposite to $N$. Define $N_{\tilde{w}}=U \cap w N^{-} w^{-1}$, where $w$ is a representative of $\tilde{w}$ in $G$. For $f \in V(\nu, \sigma, \theta)_{\infty}$, define

$$
A(\nu, \sigma, w) f(g)=\int_{N_{\tilde{w}}} f(g n w) d n \quad(g \in G)
$$

(cf. (3.1) and (3.2) of Section 3). The convergence and meromorphic continuation of $A(\nu, \sigma, w) f$ has been studied by Knapp and Stein in [12,13] (for minimal $P$ see also Schiffmann [24]).

The representation $I(\nu, \sigma, \theta)$ is $\chi$-generic. Let $\kappa(\nu, \sigma)$ be the canonical Whittaker functional attached to $V(\nu, \sigma, \theta)_{\infty}$ by relation (2.2) of Section 2. Then the local coefficient $C_{\chi}(\nu, \sigma, \theta, w)$ is a complex number defined by

$$
\kappa(\nu, \sigma)(f)=C_{\chi}(\nu, \sigma, \theta, w) \kappa(\tilde{w}(\nu), \tilde{w}(\sigma))(A(\nu, \sigma, w)(f)),
$$

$\forall f \in V(\nu, \sigma, \theta)_{\infty}$ (cf. Section 3 and [27]).

Received September 27, 1984. Revision received August 6, 1985. Partially supported by NSF grants MCS-8101600, MCS-8320317, and MCS-8108814(A02). 
To explain the significance of $C_{\chi}(\nu, \sigma, \theta, w)$, let $W_{\mathbf{R}}$ be the Weil group of $\mathbf{R}$, and if ${ }^{L} M$ is the $L$-group of $\mathbf{M}$, let $\varphi: W_{\mathbf{R}} \rightarrow{ }^{L} M$ be the homomorphism attached to $\sigma$ by Langlands' local class field theory at infinity (cf. [21]). Next, if ${ }_{\mathfrak{n}}$ is the Lie algebra of the $L$-group of $\mathbf{N}$, let ${ }^{L} \mathfrak{n}_{\tilde{w}}$ be the subspace generated by those roots $\alpha^{\vee}$ in $L_{\mathfrak{n}}$ for which $\tilde{w}(\alpha)$ is negative. Finally, let $\rho$ be the adjoint action of ${ }^{L} M$ on ${ }^{L_{\mathfrak{n}_{\tilde{w}}}}$. Then $\rho \cdot \varphi$ is a complex representation of $W_{\mathbf{R}}$. If $\rho_{\theta}$ is half the sum of the roots in $N$, we let $a_{1}, \ldots, a_{n}$ be the distinct values of $2 \rho_{\theta}\left(\alpha^{\vee}\right)$. Now, for each $i$, $i=1, \ldots, n$, let $V_{i}$ be the subspace of ${ }^{L} \mathfrak{n}_{\tilde{w}}$ generated by those $\alpha^{\vee}$ for which $2 \rho_{\theta}\left(\alpha^{\vee}\right)=a_{i}$. Then each $V_{i}$ is $W_{\mathbf{R}}$-invariant under $\rho \cdot \varphi$. Let $r_{i}=\rho \cdot \varphi \mid V_{i}$ and denote its contragredient by $\tilde{r}_{i}$ (cf. [19, 20, 27]).

Fix a complex number $s$ and let $\psi_{\mathbf{R}}(x)=\exp (2 \pi i x)$. Extend $\psi_{\mathbf{R}}$ to a character $\chi$ of $U$ as in Section 3. Now, for each $i$, let $L\left(s, r_{i}\right)$ and $\epsilon\left(s, r_{i}, \psi_{\mathbf{R}}\right)$ be the corresponding Artin $L$-function and root number, respectively. Finally, let $\lambda\left(\mathbf{C} / \mathbf{R}, \psi_{\mathbf{R}}\right)=i$ be as in [18]. The main result of this paper (Theorem 3.1) is the following:

THEOREM 1. Fix the representative $w$ of $\tilde{w}$ and the measure dn defining $A(\nu, \sigma, w)$ as in Section 3. Suppose $\sigma$ is $\chi$-generic. Then

$$
C_{\chi}\left(-2 s \rho_{\theta}, \sigma, \theta, w\right)=\lambda\left(\mathbf{C} / \mathbf{R}, \psi_{\mathbf{R}}\right)^{2 m+p} \prod_{i=1}^{n} \epsilon\left(a_{i} s, r_{i}, \psi_{\mathbf{R}}\right) \frac{L\left(1-a_{i} s, \tilde{r}_{i}\right)}{L\left(a_{i} s, r_{i}\right)}
$$

where $m$ and $p$ are the number of rank one groups $G_{\alpha}\left(\alpha\right.$ reduced $a_{0}$-root, $X_{\alpha} \in N$, and $\tilde{w}(\alpha)$ negative), whose simply connected coverings are isomorphic to $\mathrm{SU}(2,1)$ and $\mathrm{SL}(2, \mathrm{C})$, respectively.

The significance of the theorem comes from the following observation. Suppose there exists a cusp form $\pi=\bigotimes_{v} \pi_{v}$ (over a number field) such that $\sigma=\pi_{v}$ for some archimedean place $v$. Then $C_{\chi}\left(-2 s \rho_{\theta}, \pi_{v}, \theta, w_{\theta}\right)$ is the local factor (at $v$ ) which appears in the functional equation satisfied by $\prod_{i=1}^{n} L\left(a_{i} s, \pi\right.$, $r_{i}$ ) (Theorem 4.1 of [27]). Consequently, they are expected to have the form (up to the factor $\left.\lambda\left(\mathbf{C} / \mathbf{R}, \psi_{\mathbf{R}}\right)^{2 m+p}\right)$ given by the right hand side of (1) and the importance of Theorem 1 is that this is in fact true when $v$ is archimedean.

Since the statement is also true when $v$ is unramified, Theorem 4.1 of [27] now establishes the functional equation

$$
\prod_{i=1}^{n} L\left(a_{i} s, \pi, r_{i}\right)=\prod_{i=1}^{n} \epsilon\left(a_{i} s, \pi, r_{i}\right) L\left(1-a_{i} s, \pi, \tilde{r}_{i}\right),
$$

whenever infinity is the only ramification for $\pi$; in particular, (2) holds for every classical cusp form on $\mathrm{SL}_{2}$ (with respect to $\mathrm{SL}_{2}(\mathbf{Z})$; cf. [22] for an example).

Moreover, Theorem 1, together with Theorems 5.1 of [28] and 4.1 of [27] will provide us with a new proof of the functional equation satisfied by the Rankin-Selberg $L$-functions attached to the pairs of cusp forms on $\mathrm{GL}_{n}$ and $\mathrm{GL}_{m}$, as defined by Jacquet, Piatetski-Shapiro, and Shalika (cf. [7, 8]). The original proof is due to them. 
As a corollary of Theorem 1, in Section 4, we establish (Theorem 4.1) an identity satisfied by an important normalization of the operator $A(\nu, \sigma, w)$, whenever $w \sigma \cong \sigma$ (cf. [2, 9]). We refer to [1] and [29] for the significance of such identities. We only remark that as is the case with $\operatorname{SU}(2,1)$ (cf. $[9,30]$ ), it is expected that at every other ramified place, the corresponding local analogue of $\lambda\left(\mathbf{C} / \mathbf{R}, \psi_{\mathbf{R}}\right)^{2 m+p}$ will appear, so that their global product becomes 1 .

To define $C_{\chi}(\nu, \sigma, \theta, w)$, one needs the analytic continuation of $\kappa(\nu, \sigma)$ which by Proposition 2.4 reduces to that of Jacquet integrals, defined by (1.2). These integrals were first introduced by Jacquet in [6], where he established their analytic continuation for Chevalley groups. This was later extended to any rank one real group by Schiffmann [24]. Following Jacquet, in [26], we extended his result from the $K$-finite functions to the smooth ones, but still only for split groups. About the same time Hashizume [5] extended Jacquet's result to certain $K$-finite functions, but on a general reductive group. Recently, Wallach [36] has proved a general result which extends this result to any quasi-split group. This is what we choose as our reference for Theorem 1.3 of the present paper, even though the theorem can be proved along the same lines as in $[6,26]$. Now, Theorem 1.3, together with Proposition 2.4 (see also the remark at the end of Section 2), provide us with a proof of a result, already stated, but not proved, in [27] (Proposition 3.2). Finally, we would like to remark that quasi-split groups are all one needs to consider, when studying generic representations (Corollary 5.8 of [33]).

Proof of Proposition 2.4 is based on certain deep results of Casselman and Wallach, which are only partially published [34]. Fortunately, in view of a result of Vogan (part $f$ of Theorem 6.2 of [33]), we can extract what we need from Wallach's published article [34] (cf. Proposition 2.2 of the present paper).

Theorem 3.1 is proved through a number of lemmas, most of which are based on the material in Section 7 of Knapp-Zuckerman's [15]. In fact, it can easily be seen that $\sigma$ may be taken to be in the discrete series. Next, if we imbed $\sigma \subset I_{M}\left(\nu_{0}, \eta_{0}\right)$, a nonunitary principal series of $M$, we get $C_{\chi}(\nu, \sigma, \theta, w)=C_{\chi}\left(\nu_{0}+\right.$ $\left.\nu, \eta_{0}, \varnothing, w\right)$ which can now be computed by Lemma 1.4. The difficult part is to show that $C_{\chi}\left(\nu_{0}+\nu, \eta_{0}, \varnothing, w\right)$ is in fact equal to the right hand side of (1), for which one needs two basic ingredients. The first is that of understanding all the possible extensions of a-roots to $\mathfrak{a}_{0}$-roots; and the second is that of relating the parameters $\nu_{0}$ and $\eta_{0}$ to the Harish-Chandra parameter of $\sigma$. We were fortunate, since Lemmas 7.4, 7.5 and 7.6 of [15] which are in turn consequences of Knapp's earlier work $[10,11]$, were aimed exactly at answering the first problem; while the second one was the subject of study by Knapp and Wallach in [14]. The proof of Theorem 3.1 then reduces to a number of lemmas (Lemmas 3.2 through 3.12). We hope that our theorem gives another application of the deep results available in Section 7 of [15].

Theorem 4.1 is now a consequence of Theorem 3.1 and a result of Delorme [3] on the ratios of intertwining operators. I am indebted to L. Clozel for informing me of Delorme's result. I also would like to thank him for useful discussions during the past year. 
Thanks are also due to J. Arthur, D. Vogan, and N. Wallach for useful conversations.

0. Notation. Let $\mathbf{G}$ be a quasi-split connected reductive algebraic group over R. Fix a maximal torus $\mathbf{T}$ over $\mathbf{R}$, and let $\mathbf{B}$ be a fixed Borel subgroup containing $\mathbf{T}$. Then $\mathbf{B}=\mathbf{T U}$, where $\mathbf{U}$ is the unipotent radical of $\mathbf{B}$. For every algebraic group $\mathbf{H}$, we use $H$ to denote $\mathbf{H}(\mathbf{R})$. We then have $G, B, T, U, \ldots$ Let $K$ be a maximal compact subgroup of $G$ relative to $B$. Then $G=K B$.

Let $\Sigma$ denote the set of roots of $T$ in $G$. Then $U$ determines an ordering in $\Sigma$. Let $\Sigma=\Sigma^{+} \cup \Sigma^{-}$with $\Sigma^{+}$and $\Sigma^{-}$the sets of positive and negative roots in $\Sigma$, respectively. Denote by $\Delta$ the set of simple roots in $\Sigma^{+}$. We say $\alpha \in \Sigma$ is reduced if $\frac{1}{2} \alpha \notin \Sigma$.

For a subset $\theta \subset \Delta$, let $\mathbf{M}_{\theta}$ be the reductive subgroup of $\mathbf{G}$ generated by $\boldsymbol{\theta}$. Denote by $\mathbf{P}_{\theta}$ the corresponding standard parabolic subgroup, i.e., $\mathbf{P}_{\theta}=\mathbf{M}_{\theta} \mathbf{N}_{\theta}$ with $\mathbf{N}_{\theta} \subset \mathbf{U}$. Then $\mathbf{U}=\mathbf{N}_{\varnothing}$ and $\mathbf{B}=\mathbf{P}_{\varnothing}$. Let $\mathbf{A}_{\theta}$ be the split component of the center of $\mathbf{M}_{\theta}$. Write $P_{\theta}=M_{\theta}^{0} A_{\theta} N_{\theta}$ for the corresponding Langlands decomposition of $P_{\theta}$. When $\theta$ is the empty set, we use $M_{0}$ and $A_{0}$ to denote $M_{\varnothing}^{0}$ and $A_{\varnothing}$, respectively. Then $B=M_{0} A_{0} U$. Since $\mathbf{G}$ is quasi-split, $M_{0}$ is abelian. It is the centralizer of $A_{0}$ in $K$.

For every $\theta \subset \Delta$, let $W\left(A_{\theta}\right)$ be the Weyl group of $A_{\theta}$ in $G$. When $A_{\theta}=A_{0}$, we simply use $W$ to denote $W\left(A_{0}\right)$. For every reduced root $\alpha \in \Sigma^{+}$, let $\tilde{w}_{\alpha} \in W$ be the corresponding reflection and let $\tilde{w}_{l}$ be the longest element in $W$.

Let $\mathfrak{g}$ be the Lie algebra of $G$, and for $\theta \subset \Delta$, let $\mathfrak{a}_{\theta}$ be the Lie algebra of $A_{\theta}$. Denote by $\left(\mathfrak{a}_{\theta}\right)_{\mathrm{C}}^{*}$ the complex dual of $\alpha_{\theta}$. We use $a_{\mathbf{C}}^{*}$ to denote $\left(\mathfrak{a}_{\varnothing}\right)_{C}^{*}$. Let $\Sigma_{\theta}^{+}$be the subset of roots in $\Sigma^{+}$whose root vectors lie in $N_{\theta}$. By restriction, $\Sigma_{\theta}^{+}$can then be identified with the set of roots of $A_{\theta}$ in $P_{\theta}$. Set $\rho_{\theta}=\frac{1}{2} \sum_{\alpha \in \Sigma_{\theta}^{+}} \alpha$.

Given an irreducible admissible Banach (in particular unitary) representation $(\sigma, H(\sigma))$ of $M_{\theta}^{0}$ and $\nu \in\left(\mathfrak{a}_{\theta}\right)_{\mathbf{C}}^{*}$, let

$$
I(\nu, \sigma, \theta)=\operatorname{Ind}_{M_{\theta}^{0} A_{\theta} N_{\theta} \uparrow G} \sigma \otimes e^{\nu} .
$$

More precisely, this is the left inverse action of $G$ on the space $V(\nu, \sigma, \theta)$ of $H(\sigma)$-valued functions $f$ satisfying

(1) $f(x$ man $)=\sigma\left(m^{-1}\right) e^{-\left(\nu+\rho_{\theta}\right)(\log a)} f(x)$ for all $a \in A_{\theta}, \quad m \in M_{\theta}^{0}, \quad n \in N_{\theta}$, $x \in G$, and

(2) $f$ is continuous.

When $\sigma$ is a unitary representation, we shall enlarge the space by replacing (2) by the standard condition

$$
\int_{K}|f(k)|^{2} d k<\infty
$$

If $\sigma$ is a differentiable representation of $M_{\theta}^{0}$ on a Fréchet space $H(\sigma)$, we use $I(\nu, \sigma, \theta)$ for the differentiably induced representation $\operatorname{Ind}_{M_{\theta}^{0} A_{\theta} N_{\theta} \uparrow G} \sigma \otimes e^{\nu}$. The corresponding space $V(\nu, \sigma, \theta)$ is then the space of all the functions 
$C^{\infty}(G, H(\sigma))$ which satisfy relation (1) above. If $(\sigma, H(\sigma))$ is a Banach representation as above, and $\sigma_{\infty}$ is the corresponding differentiable representation on the subspace of smooth vectors, then $I\left(\nu, \sigma_{\infty}, \theta\right)=I(\nu, \sigma, \theta)_{\infty}$, the corresponding differentiable representation of $G$ on the space of smooth vectors in $V(\nu, \sigma, \theta)$ (cf. [23]).

Finally, let $\mathfrak{g}$ be the Lie algebra of $G$, and denote by $\mathfrak{H}(\mathfrak{g})$ the universal enveloping algebra of the complexification of $g$. Let $\pi$ be a continuous representation of $G$ on a Fréchet space $V$. Denote by $V_{K}$ the corresponding subspace of $K$-finite vectors. Then $\mathfrak{g}$ and consequently $\mathfrak{H}(\mathfrak{g})$ both act on $V_{K}$. Moreover if $\left(\pi_{\infty}, V_{\infty}\right)$ is the corresponding differentiable representation and $V_{\infty}$ is equipped with the Schwartz topology of $C^{\infty}(G, V)$, then $V_{K}$ is dense in $V_{\infty}$. With the above notation, $V(\nu, \sigma, \theta)_{K}$ is then the space of $K$-finite functions in $V(\nu, \sigma, \theta)$.

1. Whittaker functionals for the principal series. Throughout this section we shall only consider the principal series, i.e., those induced from $B$. Then $U=N_{\varnothing}$.

For every $\alpha \in \Sigma$, let $U_{\alpha}$ be the subgroup of $U$ whose Lie algebra is $\mathfrak{g}_{\alpha}+\mathfrak{g}_{2 \alpha}$. The subgroup $\prod_{\alpha \in \Sigma^{+}-\Delta} U_{\alpha}$ is normal in $U$ and the quotient is isomorphic to $\prod_{\alpha \in \Delta}\left(U_{\alpha} / U_{2 \alpha}\right)$. For each $\alpha \in \Delta$, let $\chi_{\alpha}$ be a continuous character of $U_{\alpha} / U_{2 \alpha}$. Then $\chi=\prod_{\alpha \in \Delta} \chi_{\alpha}$ is a character of $\prod_{\alpha \in \Delta}\left(U_{\alpha} / U_{2 \alpha}\right)$ and therefore one of $U$, and every character of $U$ is so obtained. A character of $U$ is said to be nondegenerate if it is of this form with no $\chi_{\alpha}$ trivial. Throughout this section we shall fix a nondegenerate character $\chi$ of $U$.

Now, let $(\pi, V)$ be a continuous representation of $G$ on an appropriate space (i.e., such that $V_{\infty}$ can be defined). We shall say $(\pi, V)$ is $\chi$-nondegenerate if there exists a continuous nonzero linear functional $\lambda$ on $V_{\infty}$ such that

$$
\lambda(\pi(u) v)=\chi(u) \lambda(v) \quad\left(u \in U, v \in V_{\infty}\right) .
$$

Such a $\lambda$ is called a $\chi$-Whittaker functional. Let $V_{\chi}^{*}$ be the space of all $\chi$-Whittaker functionals. Then $\operatorname{Dim}_{\mathbf{C}} V_{\chi}^{*} \leqslant 1$ if $\pi$ is irreducible and unitary [31].

Moreover, fix $\theta \subset \Delta$, and let $(\pi, V)$ be a differentiable Fréchet representation of $M_{\theta}^{0}$ and set $W=V(0, \pi, \theta)$ and $I=I(0, \pi, \theta)$. We then have (Theorem 2.2 of $[5])$.

Theorem 1.1. $\operatorname{Dim}_{\mathbf{C}} W_{\chi}^{*} \leqslant \operatorname{Dim}_{\mathbf{C}} V_{\chi}^{*}$. In particular $\operatorname{Dim}_{\mathbf{C}} W_{\chi}^{*} \leqslant 1$ if $\theta=\varnothing$.

Remark. Theorem 1.1 is clearly true, if $\sigma$ is replaced by any representation for which $V(\nu, \sigma, \theta)_{\infty}=V\left(\nu, \sigma_{\infty}, \theta\right)$. In particular, it holds for the representations induced continuously (quasi-unitarily) from a Banach (resp. unitary) representation $\sigma$ of $M_{\theta}^{0}$, as discussed before.

Now for $\tilde{w} \in W=W\left(A_{0}\right)$, let

$$
\Delta(\tilde{w})=\left\{\alpha \in \Sigma^{+} \mid \tilde{w}(\alpha) \in \Sigma^{-}\right\} .
$$

Also for $\alpha \in \Sigma^{+}$, define a coroot $H_{\alpha} \in \mathfrak{a}_{\varnothing}$ by $\alpha\left(H_{\alpha}\right)=2$, and using the notation 
in [24] set

$$
S(\tilde{w})=\left\{\nu \in \mathfrak{a}_{\mathbf{C}}^{*} \mid \operatorname{Re}\left(\nu\left(H_{\alpha}\right)\right)>0, \forall \alpha \in \Delta(w)\right\} .
$$

For the sake of simplicity we use $I(\nu, \eta)$ and $V(\nu, \eta), \nu \in \mathfrak{a}_{\mathbf{C}}^{*}, \eta \in \hat{M}_{0}$, to denote $I(\nu, \eta, \varnothing)$ and $V(\nu, \eta, \varnothing)$, respectively. The following proposition suggests a canonical choice for a basis in $V(\nu, \eta)_{\chi}^{*}$ at least when $\nu \in S\left(\tilde{w}_{l}\right)$. Its proof is trivial (cf. [26]).

Proposition 1.2. Fix $\nu \in S\left(\tilde{w}_{l}\right)$ and let $w_{l}$ be a representative for $\tilde{w}_{l}$. Then $I(\nu, \eta)$ is $\chi$-generic and a $\chi$-Whittaker functional for $V(\nu, \eta)$ is given by

$$
\lambda(\nu, \eta)(f)=\int_{U} f\left(u w_{l}\right) \chi(u) d u \quad f \in V(\nu, \eta)_{\infty} .
$$

Again as in [26], let $G_{D}$ be the derived group of $G$. Denote by $\tilde{G}_{D}$ the simply connected covering of $G_{D}$. There exists a homomorphism $\alpha$ from $\tilde{G}_{D}$ into $G_{D}$ which is an isomorphism on $U$. For a function $f \in V(\nu, \eta)_{\infty}$, we define a function $\tilde{f}$ on $\tilde{G}_{D}$ by $\tilde{f}(g)=f(\alpha(g))$. Then $\tilde{f}$ belongs to a principal series representation of $\tilde{G}_{D}$. Let $\tilde{\lambda}$ be the $\chi$-Whittaker functional for this principal series of $\tilde{G}_{D}$ defined by (1.2). Then $\lambda(f)=\tilde{\lambda}(\tilde{f}), f \in V(\nu, \eta)_{\infty}$, where $\lambda=\lambda(\nu, \eta)$. Consequently study of Whittaker functionals reduces to those for simply connected semisimple groups.

Now, given $\varphi \in C_{c}^{\infty}(G)$, define

$$
f_{\varphi}(g, \nu, \eta)=\int_{M_{0} \times A_{0} \times U} \eta\left(m_{0}\right) e^{(\nu+\rho)(\log a)} \varphi\left(g m_{0} a u\right) d m_{0} d a d u .
$$

Then $f_{\varphi} \in V(\nu, \eta)_{\infty}$. Now for $\nu \in S\left(\tilde{w}_{l}\right)$, set

$$
W_{\varphi}(g, \nu, \eta)=\int_{U} f_{\varphi}\left(g u w_{l}, \nu, \eta\right) \chi(u) d u .
$$

Then $\lambda(\nu, \eta)\left(f_{\varphi}\right)=W_{\varphi}(e, \nu, \eta)$. The following result can be proved along the same lines as in $[6,26]$ (already proved for split groups). But since a more general result is already available [36], we shall only state the result and refer the reader to [36] for the proof.

THEOREM 1.3. Fix $\varphi \in C_{c}^{\infty}(G)$. Then $W_{\varphi}(g, \nu, \eta)$ extends to an entire function of $\nu$ on $a_{\mathbf{C}}^{*}$.

COROLlaRy. The analytic continuation of $\lambda(\nu, \eta)$ defines a Whittaker functional for each $I(\nu, \eta), \nu \in \mathfrak{a}_{\mathbf{C}}^{*}$ and $\eta \in \hat{M}_{0}$.

To conclude this section, we shall state and prove a lemma (Lemma 1.4) which we need later.

Assume that $G$ is of semisimple rank one. Then there are three possibilities.

1. $\tilde{G}_{D}=\mathrm{SL}_{2}(F), F=\mathbf{R}$ or $\mathbf{C}$, and

2. $\tilde{G}_{D}=\operatorname{SU}(2,1)$.

Let $\alpha$ be the reduced root in $\Sigma^{+}$, and denote by $\tilde{w}$ the nontrivial element of $W$. 
We let

$$
w=\left(\begin{array}{rr}
0 & -1 \\
1 & 0
\end{array}\right) \quad \text { if } \quad \tilde{G}_{D}=\operatorname{SL}_{2}(F), \quad F=\mathbf{R} \text { or } \mathbf{C}
$$

and

$$
w=\left(\begin{array}{rrr}
0 & 0 & 1 \\
0 & -1 & 0 \\
1 & 0 & 0
\end{array}\right) \quad \text { if } \quad \tilde{G}_{D}=\operatorname{SU}(2,1)
$$

To define $\chi=\chi_{\alpha}, \Delta=\{\alpha\}$, we fix a nontrivial additive character $\psi_{\mathbf{R}}$ of $\mathbf{R}$, and if $\alpha$ is complex we define a nontrivial character of $\mathbf{C}$ by $\psi_{\mathbf{C}}=\psi_{\mathbf{R}} \cdot \operatorname{Tr}_{\mathbf{C} / \mathbf{R}}$ $\left(\operatorname{Tr}_{\mathbf{C} / \mathbf{R}}(z)=2 \operatorname{Re}(z), z \in \mathbf{C}\right)$. We then set $\chi=\psi_{\mathbf{R}}$ or $\psi_{\mathbf{C}}$ according as $\alpha$ is real or complex.

Fix an irreducible unitary representation $\sigma$ of $K$ on the Hilbert space $H_{\sigma}$. Denote by $P(\sigma)$, the projection operator on the subspace of vectors $v \in H_{\sigma}$ satisfying

$$
\sigma(m) v=\eta\left(m^{-1}\right) v \quad\left(m \in M_{0}\right) .
$$

Consider the function $f_{\nu, \eta, \sigma}$ on $G$ defined by

$$
f_{\nu, \eta, \sigma}(k a m u)=\sigma(k) \eta\left(m^{-1}\right) e^{-(\nu+\rho)(\log a)} P(\sigma) .
$$

Let $V$ be the unipotent radical of the parabolic subgroup opposed to $B$. For $v \in V$, define $\chi^{\prime}(v)=\chi\left(w v w^{-1}\right)$. For $\nu \in S(\tilde{w})$, set

$$
T(\nu, \eta, \sigma)=\int_{V} \sigma\left(w k_{v}\right) \eta\left(m_{v}^{-1}\right) e^{-(\nu+\rho)\left(\log a_{v}\right)} P(\sigma) d v
$$

where $v=k_{v} a_{v} m_{v} u_{v}$. Then Proposition 3.2 of [24] implies that

$$
f_{w \nu, w \eta, \sigma} \cdot T(\nu, \eta, \sigma)=f_{\nu, \eta, \sigma} * \Phi_{\nu, \eta, w}
$$

where $\Phi_{\nu, \eta, w}$ is the measure defined in Paragraph 1.8 of [24]. Now, we define a Whittaker function of type $\sigma$ by

$$
W_{\sigma}(g, v, \eta)=\int_{V} f_{\nu, \eta, \sigma}(g v) \chi^{\prime}(v) d v
$$

Then by Theorem 3.3 of [24]

$$
W_{\sigma}(g, w \nu, w \eta) T(\nu, \eta, \sigma)=W_{\sigma}(g, \nu, \eta) \chi^{\prime}\left(\Phi_{\nu, \eta, w}\right) .
$$

Here $\chi^{\prime}\left(\Phi_{\nu, \eta, w}\right)$ is the Fourier transform of $\Phi_{\nu, \eta, w}$ with respect to $\chi^{\prime}$.

Now, write $\sigma \mid M_{0}=\bigoplus_{i} m_{i} \eta_{i}$, with positive integers $m_{i}$. Suppose $\eta_{1}=\eta$. Choose $e$ and $e^{\prime}$ in the space of $\eta_{1}$ and define

$$
f_{e, e^{\prime}, \nu, \eta}(k a m u)=\eta\left(m^{-1}\right) e^{-(\nu+\rho)(\log a)}\left(e \mid \sigma(k) e^{\prime}\right),
$$


where (|) denotes the inner product in $H_{\sigma}$. Then

$$
A(\nu, \eta, w) f_{e, e^{\prime}, \nu, \eta}=f_{e, T(\nu, \eta, \sigma) e^{\prime}, w v, w \eta}
$$

and (1.5) implies

$$
W_{A(\nu, \eta, w) f}(g, w v, w \eta)=\chi^{\prime}\left(\Phi_{\nu, \eta, w}\right) W_{f}(g, \nu, \eta),
$$

where by relation (3.4.3) of [24], $\chi^{\prime}\left(\Phi_{\nu, \eta, w}\right)$ is defined by

$$
\begin{aligned}
\chi^{\prime}\left(\Phi_{\nu, \eta, w}\right) & =\int_{V} \chi^{\prime}(v) d \Phi_{\nu, \eta, w}(v) \\
& =\int_{V} \chi^{\prime}(v) \eta\left(m_{v}\right) N(v)^{\nu-\rho} d v
\end{aligned}
$$

with $v=k_{v} a_{v} m_{v} u_{v}$ and $f=f_{e, e^{\prime}, v, \eta}$, at first formally. Observe that $\chi^{\prime}\left(\Phi_{v, \eta, w}\right)$ is a scalar. The intertwining operator $A(\nu, \eta, w)$ for each $\nu \in S(\tilde{w})$ is defined by

$$
A(\nu, \eta, w) f(g)=\int_{U} f(g u w) d u \quad\left(f \in V(\nu, \eta)_{\infty}\right) .
$$

The measure $d u$ is normalized according to Tate's thesis, i.e., it is the ordinary Lebesgue measure on the root space of a real root and is equal to $i d z \wedge d \bar{z}$ on the root space of a complex root. Fix $\psi_{\mathbf{R}}$ by $\psi_{\mathbf{R}}(x)=\exp (2 \pi i x), x \in \mathbf{R}$. Finally, fix $H_{\alpha} \in$ a such that $\alpha\left(H_{\alpha}\right)=2$. Denote $\nu\left(H_{\alpha}\right)$ by $\nu$ as well. To be in agreement with the notation in [27], let $C_{\chi}^{-1}(\nu, \eta, \varnothing, w)$ denote $\chi^{\prime}\left(\Phi_{\nu, \eta, w}\right)$.

Lemma 1.4. Fix $\chi$, the representative $w$ and the measure du defining $A(\nu, \eta, w)$ (and consequently $C_{\chi}(\nu, \eta, \varnothing, w)$ ) as above.

(a) Suppose $\tilde{G}_{D}=\mathrm{SL}_{2}(F)$, where $F=\mathbf{R}$ or $\mathbf{C}$. Then

$$
C_{\chi}(\nu, \eta, \emptyset, w)=\gamma(\eta, \nu)
$$

where $\gamma(\eta, \nu)$ is the corresponding local Tate coefficient. More precisely, if $F=\mathbf{R}$ and $\eta(m)=\operatorname{sgn}(m)^{\epsilon}, \epsilon=0,1$, then

$$
\gamma(\eta, \nu)=i^{\epsilon} \frac{\pi^{-1 / 2(\epsilon+1-\nu)} \Gamma(1 / 2(\epsilon+1-\nu))}{\pi^{-1 / 2(\epsilon+\nu)} \Gamma(1 / 2(\epsilon+\nu))}
$$

and if $F=\mathbf{C}$, and

$$
\eta\left(\left(\begin{array}{cc}
e^{i \theta} & 0 \\
0 & e^{-i \theta}
\end{array}\right)\right)=e^{i n \theta} \quad n \in \mathbf{Z}
$$

then

$$
\gamma(\eta, \nu)=i^{|n|} \frac{2(2 \pi)^{-(1-\nu / 2)} \Gamma(1-\nu / 2+|n| / 2)}{2(2 \pi)^{-\nu / 2} \Gamma(\nu / 2+|n| / 2)}
$$


(b) Suppose $\tilde{G}_{D}=\mathrm{SU}(2,1)$. If

$$
\eta\left(\left(\begin{array}{ccc}
u & 0 & 0 \\
0 & u^{-2} & 0 \\
0 & 0 & u
\end{array}\right)\right)=u^{n} \quad n \in \mathbf{Z}
$$

then

$$
C_{\chi}(\nu, \eta, \varnothing, w)=(2 \pi)^{\nu-2} \frac{\Gamma(1-\nu / 4+n / 2) \Gamma(1-\nu / 4-n / 2)}{\Gamma(\nu / 2)} .
$$

Proof. By Lemma 4.4 of [25], we only need to prove part (b). Given $v \in V$, choose $X \in \mathfrak{g}_{-\alpha}$ and $Y \in \mathfrak{g}_{-2 \alpha} \cong i \mathbf{R}$ such that $v=\exp \left(X+\frac{1}{2} Y\right)$. Write $w v$ $\in V m_{v} a_{v} U$, with $m_{v}=\operatorname{diag}\left(u, u^{-2}, u\right) \in M_{0}$ and $a_{v}=\operatorname{diag}\left(e^{t}, 1, e^{-t}\right) \in A_{0}$. Then using the formulas in page 556 of [12], we have

$$
u=\frac{\|X\|^{2}+Y}{\left(\|X\|^{4}+|Y|^{2}\right)^{1 / 2}}
$$

and

$$
e^{t}=\frac{1}{2}\left(\|X\|^{4}+|Y|^{2}\right)^{1 / 2}
$$

where \|\| and | | are, respectively, the standard norms on $\mathfrak{g}_{\alpha} \cong \mathbf{R}^{2}$ and $\mathfrak{g}_{2 \alpha} \cong i \mathbf{R}$, $i=\sqrt{-1}$. Moreover $N(v)=\left\langle 1, a_{v}\right\rangle$ in relation (1.7) is given by

$$
N(v)=\frac{1}{\sqrt{2}}\left(\|X\|^{4}+|Y|^{2}\right)^{1 / 4}
$$

Using $\rho=4$, and $d v=d X d(-i Y / 2),(1.7)$ is again formally equal to $2^{-\nu / 2+1} \int_{\mathbf{R}^{2} \times i \mathbf{R}}\left(\|X\|^{2}+Y\right)^{n}\left(\|X\|^{4}+|Y|^{2}\right)^{1 / 4(\nu-2 n-4)} \psi_{\mathbf{C}}(X) d X d(-i Y)$.

Set $T(v)=\left(\|X\|^{4}+|Y|^{2}\right)^{1 / 4}$. Then, using (cf. [24])

$$
T(v)^{\nu-2 n-4}=\frac{8}{\Gamma(1 / 4(4+2 n-\nu))} \int_{A_{0}}\langle 2 \nu-8-4 n, a\rangle e^{-T\left(a v a^{-1}\right) / 4} d a,
$$

and changing $v$ to $a^{-1} v a,(1.4 .1)$ is equal to

$$
\frac{8 \cdot 2^{-v / 2+1}}{\Gamma(1 / 4(4+2 n-v))} \int_{0}^{+\infty} t^{2 \nu} \int_{V}\left(\|X\|^{2}+y\right)^{n} e^{-T(v)^{4}} \chi^{\prime}\left(a_{t}^{-1} v a_{t}\right) d v d t
$$

where $a_{t}^{-1} v a_{t}=\exp \left(t^{2} X+t^{4} Y\right)$, and $\operatorname{Re}(\nu)>0$ (for $t>0, a_{t} \in A_{0}$ is the unique element defined by $t=\left\langle 1, a_{t}\right\rangle$ in the notation of [24]). It is in this sense, i.e., as a 
Mellin transform, that (1.7) is defined as a convergent integral (cf. Proposition 3.3 of [24]). Of course, one must stay away from the singularities of the $\Gamma$-function. Set

$$
I_{1}=\int_{0}^{+\infty} t^{2 \nu} \int_{\substack{X \in \mathbf{R}^{2}-\{0\} \\ Y \in i \mathbf{R}}}\left(\|X\|^{2}+Y\right)^{n} \psi_{\mathbf{C}}\left(t^{2} X\right) \exp \left(-\|X\|^{4}-|Y|^{2}\right) d^{*} t d X d(-i Y)
$$

Then (1.4.2) is equal to

$$
\frac{8 \cdot 2^{-\nu / 2+1}}{\Gamma(1 / 4(4+2 n-\nu))} I_{1}
$$

Now, if we first change $Y$ to $\|X\|^{2} Y$ and then $X$ to $X /\left(1+|Y|^{2}\right)^{1 / 4}$, we get

$$
\begin{aligned}
I_{1}= & \int_{0}^{+\infty} t^{2 v} \int_{\substack{X \in \mathbf{R}^{2}-\{0\} \\
Y \in i \mathbf{R}}}\|X\|^{2 n+2}(1+Y)^{n}\left(1+|Y|^{2}\right)^{-n / 2-1} \\
& \cdot \exp \left(-\|X\|^{4}\right) \psi_{\mathbf{C}}\left(t^{2} X /\left(1+|Y|^{2}\right)^{1 / 4}\right) d X d(-i Y) d^{*} t
\end{aligned}
$$

Finally, changing $t^{2}$ to $t, t$ to $t /\left(1+|Y|^{2}\right)^{1 / 4}$, and $t$ to $t^{2}$, we have

$$
I_{1}=\int_{0}^{+\infty} t^{2 v} \int_{X \in \mathbf{R}^{2}}\|X\|^{2 n+2} \psi_{\mathbf{C}}\left(t^{2} X\right) \exp \left(-\|X\|^{4}\right) d X d^{*} t . I_{2},
$$

where

$$
\begin{aligned}
I_{2} & =\int_{Y \in i \mathbf{R}}(1+Y)^{n}\left(1+|Y|^{2}\right)^{-n / 2-1+\nu / 4} d(-i Y) \\
& =\int_{-\infty}^{+\infty}\left(\frac{1+i t}{1-i t}\right)^{n / 2}\left(1+t^{2}\right)^{-1+\nu / 4} d t .
\end{aligned}
$$

Now setting $t=\tan \theta$, we see that for $\operatorname{Re}(\nu)<2$

$$
\begin{aligned}
I_{2} & =2 \int_{0}^{\pi / 2} \cos n \theta \cos ^{-\nu / 2} \theta d \theta \\
& =\frac{2 \pi \Gamma(1-\nu / 2)}{2^{-\nu / 2+1} \Gamma(1-\nu / 4+n / 2) \Gamma(1-\nu / 4-n / 2)},
\end{aligned}
$$

using an exercise in [35].

Consequently for $0<\operatorname{Re}(v)<2, I_{1}$ is convergent, and to compute it, we only need to evaluate

$$
I_{3}=\int_{0}^{+\infty} t^{2 \nu} \int_{X \in \mathbf{R}^{2}}\|X\|^{2 n+2} \psi_{\mathbf{C}}\left(t^{2} X\right) \exp \left(-\|X\|^{4}\right) d X d^{*} t
$$


We realize $\mathbf{R}^{2} \cong \mathbf{C}$ and define the absolute value || on $\mathbf{C}$ by $|X|=\|X\|^{2}=r^{2}$ ( $r$ is the polar radius). Now define a function $f$ on $\mathbf{C}$ by $f(r, \theta)=r^{2 n+2} e^{-r^{4}}$. We denote the realization of $f$ on $\mathbf{R}^{2}$ by $g(x, y)$, where $x^{2}+y^{2}=r^{2}$. Then the integral over $\mathbf{R}^{2}$ is equal to $\hat{g}\left(t^{2}, t^{2}\right)$, the Fourier transform of $g$ on $\mathbf{R}^{2}$. Consequently

$$
\begin{aligned}
I_{3} & =\int_{0}^{+\infty} t^{2 v} \hat{g}\left(t^{2}, t^{2}\right) d^{*} t \\
& =\frac{1}{8 \pi} \int_{\mathbf{C}}|\alpha|^{\nu / 2} \hat{g}(\alpha) d^{*} \alpha .
\end{aligned}
$$

Applying Tate's local (complex) functional equation to (1.4.3), we get

$$
I_{3}=\frac{1}{8 \pi} \cdot \frac{2 \cdot(2 \pi)^{-\nu / 2} \Gamma(\nu / 2)}{2 \cdot(2 \pi)^{-(1-\nu / 2)} \Gamma(1-\nu / 2)} \int_{\mathbf{C}}|\alpha|^{1-\nu / 2} g(\alpha) d^{*} \alpha .
$$

Next, if we substitute $|\alpha|=r^{2}, d^{*} \alpha=2 r d r d \theta / r^{2}$, and $g(\alpha)=r^{2 n+2} e^{-r^{4}}$, we see that

$$
I_{3}=\frac{1}{8} \cdot \frac{(2 \pi)^{-\nu / 2} \Gamma(\nu / 2)}{(2 \pi)^{-(1-\nu / 2)} \Gamma(1-\nu / 2)} \Gamma(1 / 4(4-\nu+2 n)) .
$$

This completes the lemma.

Remark 1. As we shall discuss in Section 3, the exact value of $C_{\chi}(\nu, \eta, \varnothing, w)$ is desired since these factors play an important arithmetic role by appearing in the functional equation satisfied by certain $L$-functions [25, 27].

Remark 2. The formula in Theorem 3.2.2 of [27] needs an extra factor $2^{q_{\beta}\left(1 / 2\left\langle\nu_{0}+\tilde{\nu}, H_{\beta}\right\rangle-1\right)}$ which we hereby correct.

2. Whittaker functionals for induced representations. Let $g_{\mathbf{C}}$ be the complexification of $\mathfrak{g}$ and denote by $G_{\mathbf{C}}$ a connected complex group whose Lie algebra is $\mathfrak{g}_{\mathbf{C}}$. As in [16], let

$$
G_{\text {Max }}=\left\{g \in G_{\mathbf{C}} \mid(a d g) \mathfrak{g}=\mathfrak{g}\right\} .
$$

Let $T_{\mathbf{C}}$ be a maximal torus of $G_{\mathbf{C}}$ such that $T=T_{\mathbf{C}} \cap G$. We set $T_{\mathrm{Max}}=T_{\mathrm{C}} \cap G_{\mathrm{Max}}$. Then $T_{\mathrm{Max}}=M_{\mathrm{Max}} A_{0}$ and every $\eta \in \hat{M}_{0}$ extends to $\tilde{\eta}$ $\in \hat{M}_{\text {Max }}$ and moreover $I(\nu, \tilde{\eta}) \mid G=I(\nu, \eta), \nu \in \mathfrak{a}_{\mathbf{C}}^{*}$. Assume $\nu \in S\left(\tilde{w}_{l}\right)$ and let $V_{\text {Max }}$ be the unique generic subrepresentation of $V(\nu, \tilde{\eta})$ (cf. [33]). Then by Lemma 6.2.1 of [16] it is $\chi$-generic with respect to any generic character $\chi$ of $U$. Moreover $V_{\text {Max }} \mid G$ is the direct sum of all the large subrepresentations of $V(\nu, \eta)_{\infty}$, each appearing with multiplicity one [33]. Write $V_{\text {Max }} \mid G=\bigoplus_{i=1}^{n} V_{i}$ and assume $V_{1}$ has a $\chi$-Whittaker model which exists by Lemma 6.2.1 of [16]. By Corollary 6.7 of [33] choose $a_{1}=1, a_{2}, \ldots, a_{n} \in T_{\mathrm{Max}}$ (in fact, in $F_{\mathrm{Max}}$ which is defined before Theorem 6.7.2 of [16]) such that $V_{i}=V_{1}^{a_{i}}$, where for every 
representation $(\pi, H)$, we use $\left(\pi^{a}, H^{a}\right)$ to denote $\pi^{a}(g)=\pi\left(a^{-1} g a\right)$. Then $V_{i}$ is

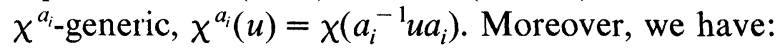

Lemma 2.1. Let $S_{\mathrm{Max}}$ be the stabilizer of $V_{1}$ in $T_{\mathrm{Max}}$. Fix $a \in T_{\mathrm{Max}}$ and assume $V_{1}$ is $\chi^{a}$-generic. Then $a \in S_{\mathrm{Max}}$. Consequently each $V_{i}$ is the unique $\chi^{a_{i} \text {-generic }}$ subrepresentation of $I(\nu, \eta)$.

Proof. We use an argument similar to the proof of Corollary 2.7 of [17]. By assumption $V_{1}$ and $V_{1}^{a^{-1}}$ are both subrepresentations of $\operatorname{Ind}_{U \uparrow G} \chi$ (differentiably induced). If they are equivalent $\operatorname{Ind}_{G \uparrow G_{\mathrm{Max}}} \bar{V}_{1}$ and $\operatorname{Ind}_{G \uparrow G_{\operatorname{Max}}} \bar{V}_{1}^{a^{-1}}$ both appear in Ind $_{U \uparrow G_{\text {Max }}} \chi$ and consequently by Frobenius reciprocity $V_{\text {Max }}$ has multiplicity at least two in $\operatorname{Ind}_{U \uparrow G_{\mathrm{Max}}} \chi$, a contradiction $\left(\bar{V}_{1}\right.$ is the closure of $V_{1}$ in $\left.V(\nu, \eta)_{\infty}\right)$.

Now, let $\theta \subset \Delta$ and fix an irreducible admissible $\chi$-generic (more precisely $\chi \mid M_{\theta} \cap U$-generic) Banach representation $(\sigma, H(\sigma))$ of $M=M_{\theta}^{0}$. Besides unitary representations, this may, for example, be taken to be the Hilbert space completion of the Langlands quotient for $H(\sigma)_{K}$ which is equal to the corresponding induced representation by part $\mathrm{f}$ of Theorem 6.2 of [33]. By Casselman's subrepresentation theorem and [33], choose $\nu_{0} \in\left(\mathfrak{a}_{M}\right)_{\mathrm{C}}^{*}$ and $\eta_{0} \in \hat{M}_{0}$ (we are assuming $A M \supset T$ and $\left.\mathfrak{a}_{0}=\mathfrak{a}_{\theta} \oplus \mathfrak{a}_{M}\right)$ with $\operatorname{Re}\left(\nu_{0}\left(H_{\alpha}\right)\right) \geqslant 0$ for all positive roots $\alpha$ in $M_{\theta}$, such that $\delta: H(\sigma)_{K} \hookrightarrow V_{M}\left(\nu_{0}, \eta_{0}\right)_{K}$, where $V_{M}\left(\nu_{0}, \eta_{0}\right)$ is the space of the representation of $M$ induced from $A_{M} M_{0}(U \cap M), A_{M}=\operatorname{expa} a_{M}$. For the sake of simplicity, let us denote the image of $H(\sigma)_{K}$ under $\delta$ again by $H(\sigma)_{K}$. Let $\overline{H(\sigma)}_{K}$ be the closure of $H(\sigma)_{K}$ in $V_{M}\left(\nu_{0}, \eta_{0}\right)_{\infty}$. We need the following proposition. It is a consequence of Casselman-Wallach's deep results [34].

Proposition 2.2. The Frechet space of differentiable vectors $H(\sigma)_{\infty} \subset H(\sigma)$ is topologically isomorphic (as a smooth M-module) to $\overline{H(\sigma)}_{K}$.

Proof. Since $\sigma$ is large [33], by part $\mathrm{f}$ of Theorem 6.2 of [33], there exists a parabolic subgroup $P^{\prime}=M^{\prime} A^{\prime} N^{\prime} \subseteq M$, with $P^{\prime} \supset B \cap M, A^{\prime}=A_{\theta^{\prime}} \cap M, \theta^{\prime} \subset \theta$, an irreducible tempered large (Corollary 6.6 of [33]) representation $\left(\sigma^{\prime}, H\left(\sigma^{\prime}\right)\right)$ of $M^{\prime}$, and $\nu^{\prime} \in\left(\mathfrak{a}^{\prime}\right)_{\mathrm{C}}^{*}$ such that $\sigma=I_{M}\left(\nu^{\prime}, \sigma^{\prime}, \theta^{\prime}\right)$, infinitesimally. For every irreducible admissible ( $\mathrm{m}, M \cap K)$-module $V$, let $\overline{\bar{V}}$ be the minimal completion of $V$ as defined in Section 6 of [34]. We use similar notation when working with $\left(\mathrm{m}^{\prime}, M^{\prime} \cap K\right)$-modules. Since $\sigma^{\prime}$ is tempered, Proposition 6.10 of [34] implies that

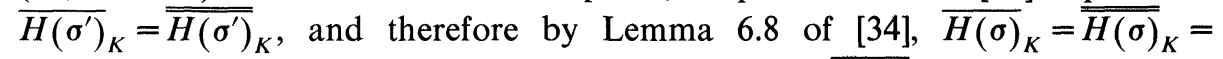
$V_{M}\left(\nu^{\prime}, \sigma^{\prime}, \theta^{\prime}\right)_{\infty}$. But then Proposition 6.12 implies that $\overline{H(\sigma)}_{K}=H(\sigma)_{\infty}=V_{M}\left(\nu^{\prime}\right.$, $\left.\sigma^{\prime}, \theta^{\prime}\right)_{\infty}$

Remark 1. By Theorem 1.1, the representation $\sigma^{\prime}$ which at first is only large must in fact be $\chi$-generic.

Remark 2. Proposition 2.2 is expected to be true for any irreducible admissible Banach representation (not only the large ones) of $M$. This follows from a result of Casselman which has not yet been even announced as a theorem 
(cf. [34]). We can only refer the reader to a Wallach's remark (Remark 6.16) in [34].

Corollary. Let $(\sigma, H(\sigma))$ be an irreducible admissible $\chi$-generic Banach representation of $M$. Then $\operatorname{Dim}_{\mathbf{C}} H(\sigma)_{x}^{*}=1$.

Proof. We only have to identify $H(\sigma)_{\infty}=\overline{\overline{H(\sigma)}}_{K}$ as a quotient of a nonunitary principal series (Proposition 6.5 of [34]).

Let $V=\delta(H(\sigma))$ and extend $\delta$ to a $G$-isomorphism $\bar{\delta}$ between $H(\sigma)_{\infty}$ and $\bar{V}$. Then $\lambda\left(\nu_{0}, \eta_{0}\right) \mid \bar{V} \not \equiv 0$ (Corollary to Theorem 1.3) and therefore $\lambda\left(\nu_{0}, \eta_{0}\right) \cdot \bar{\delta}$ is a nonzero continuous Whittaker functional for $(\sigma, H(\sigma))$. Set

$$
\lambda=\lambda\left(\nu_{0}, \eta_{0}\right) \cdot \bar{\delta} .
$$

Now, let $\tilde{w}_{l, \theta}$ be the longest element in the Weyl group $W\left(A_{M}\right)=W\left(M, A_{M}\right)$. Let $\tilde{w}_{\theta}=\tilde{w}_{l} \cdot \tilde{w}_{l, \theta}$ and choose an appropriate representative $w_{\theta}$ in $G$ for $\tilde{w}_{\theta}$, i.e., if $w_{l}$ and $w_{l, \theta}$ are the representatives of $\tilde{w}_{l}$ and $\tilde{w}_{l, \theta}$ in $G$ and $M$, fixed as in Proposition 1.2, respectively, then $w_{\theta}=w_{l} \cdot w_{l, \theta}$. Consider the induced representation $I\left(\nu, \sigma_{\infty}, \theta\right)=I(\nu, \sigma, \theta)_{\infty}, \nu \in\left(\mathfrak{a}_{\theta}\right)_{\mathrm{C}}^{*}$. Set $N^{\prime}=N_{w_{\theta}(\theta)}$. We then have:

Lemma 2.3. Take $f \in V(\nu, \sigma, \theta)_{\infty}$. Then

$$
\int_{N^{\prime}} \lambda\left(f\left(n^{\prime} w_{\theta}\right)\right) \chi\left(n^{\prime}\right) d n^{\prime}
$$

is absolutely convergent for $\operatorname{Re}\left(\nu\left(H_{\alpha}\right)\right) \gg 0, \alpha \in \Sigma_{\theta}^{+}$. In particular for such $\nu,(2.2)$ is a continuous Whittaker functional on $V(\nu, \sigma, \theta)_{\infty}$.

Proof. Let \|\| be the norm on $H(\sigma)$. Given $v \in H(\sigma)_{\infty}$, let $\tilde{v} \in C^{\infty}(M$, $\left.H(\sigma)_{\infty}\right)$ be the map $m \mapsto \sigma_{\infty}(m) v$. Then $v \mapsto \tilde{v}$ identifies $H(\sigma)_{\infty}$ as a topological subspace of $C^{\infty}\left(M, H(\sigma)_{\infty}\right)$. Let $\mathfrak{m}_{\mathrm{C}}$ be the complexification of the Lie algebra $\mathrm{m}$ of $M$. Given $D \in \mathfrak{U}\left(\mathfrak{m}_{\mathrm{C}}\right)$ and a compact set $\omega \subset M$, let $\|v\|_{D, \omega}=\sup _{x \in \omega}\|D \tilde{v}(x)\|$. By continuity of $\lambda$, there exist $D_{i} \in \mathfrak{H}\left(\mathfrak{m}_{\mathfrak{C}}\right)$ and compact subsets $\omega_{i} \subset M$, $i=1, \ldots, r$, such that

$$
|\lambda(v)| \leqslant \sum_{i=1}^{r}\|v\|_{D_{i}, \omega_{i}}
$$

Given $g \in G$, write $g=k(g) m(g) a(g) n(g)$. The set $\{\tilde{v} \mid v \in f(K)\}$ is compact in $C^{\infty}\left(M, H(\sigma)_{\infty}\right)$ and by continuity of $\varphi \mapsto\|\varphi\|_{D_{i} \omega_{i}}$ from $C^{\infty}\left(M, H(\sigma)_{\infty}\right)$ to $\mathbf{C}$ one concludes that there exists a positive constant $C_{\lambda}(f)$ such that for all $g \in G$

$$
\left\|\sigma_{\infty}(m(g)) f(k(g))\right\|_{D_{i} \omega_{i}} \leqslant C_{\lambda}(f) \quad i=1, \ldots, r .
$$

Consequently the absolute value of (2.3) is dominated by

$$
r C_{\lambda}(f) \int_{N^{\prime}}\left|e^{-\left(\nu+\rho_{\theta}\right)\left(\log \left(a\left(n^{\prime} w_{\theta}\right)\right)\right)}\right| d n^{\prime}
$$


which is finite if we choose $\operatorname{Re}\left(\nu\left(H_{\alpha}\right)\right), \alpha \in \Sigma_{\theta}^{+}$, sufficiently large (Lemma 6.5 of [13]). This completes the lemma.

Now for $\nu \in\left(\mathfrak{a}_{\theta}\right)_{\mathrm{C}}^{*}$ with $\operatorname{Re}\left(\nu\left(H_{\alpha}\right)\right) \gg 0$, set

$$
\kappa(\nu, \sigma)(f)=\int_{N^{\prime}} \lambda\left(f\left(n^{\prime} w_{\theta}\right)\right) \chi\left(n^{\prime}\right) d n^{\prime},
$$

where $f \in V(\nu, \sigma, \theta)_{\infty}$.

Let $V=\delta(H(\sigma)) \hookrightarrow V_{M}\left(\nu_{0}, \eta_{0}\right)_{K}$. Then $\bar{V}$ is $\chi$-generic. Moreover if we realize $V$ as a quotient of a principal series, Lemma 2.1 and Theorem 1.1 (applied to the complement of this quotient which is $\chi$-degenerate) shall imply that $\operatorname{Ind}_{P \uparrow G} \bar{V} \otimes$ $e^{\nu}$ is $\chi$-generic. Consequently $\lambda\left(\nu+\nu_{0}, \eta_{0}\right)$. Ind $\bar{\delta}$ defines a nonzero $\chi$-Whittaker functional for $I(\nu, \sigma, \theta)$. We shall now prove

Proposition 2.4. Suppose $\operatorname{Re}\left(\nu\left(H_{\alpha}\right)\right) \gg 0, \alpha \in \Sigma_{\theta}^{+}$. Then

$$
\kappa(\nu, \sigma)=\lambda\left(\nu+\nu_{0}, \eta_{0}\right) \cdot \operatorname{Ind} \bar{\delta}
$$

Proof. The equality (2.3) certainly holds if $\operatorname{Re}\left(\nu_{0}\left(H_{\alpha}\right)\right)>0$ for all the positive roots $\alpha \in M_{\theta}$. In fact by Theorem 1.1 , choose $C_{\sigma}\left(\nu, \nu_{0}, \eta_{0}\right) \in \mathbf{C} \cup\{\infty\}$ such that

$$
C_{\sigma}\left(\nu, \nu_{0}, \eta_{0}\right) \kappa(\nu, \sigma)=\lambda\left(\nu+\nu_{0}, \eta_{0}\right) \cdot \text { Ind } \bar{\delta} .
$$

Then $C_{\sigma}\left(\nu, \nu_{0}, \eta_{0}\right)=1$ for $\nu+\nu_{0} \in S\left(\tilde{w}_{l}\right)$.

Given $\varphi \in C_{c}^{\infty}\left(G, H(\sigma)_{\infty}\right)$, define $f_{\varphi} \in V(\nu, \sigma, \theta)_{\infty}$ by

$$
f_{\varphi}(g)=\int_{M \times A \times N} e^{\left(\nu+\rho_{\theta}\right)(\log a)} \sigma_{\infty}(m) \varphi(\text { gman }) d m d a d n .
$$

We now choose a special $\varphi$ as follows. Fix $v \in H(\sigma)_{\infty}$ and let $\bar{\varphi}$ be a complex valued function of compact support in $N^{\prime} w_{\theta} P$ of the form $\bar{\varphi}(g)=\varphi_{1}(p) \varphi_{2}\left(n^{\prime}\right)$ if $g=n^{\prime} w_{\theta} p$ and $\bar{\varphi}(g)=0$ otherwise. Here $\varphi_{1} \in C_{c}^{\infty}(P)$ and $\varphi_{2} \in C_{c}^{\infty}\left(N^{\prime}\right)$. Set $\varphi(g)=\bar{\varphi}(g) v$. Moreover suppose $\varphi_{1}($ man $)=\varphi_{1}^{\prime}(m a) \varphi_{1}^{\prime \prime}(n)$ with $\varphi_{1}^{\prime} \in C_{c}^{\infty}(M A)$ and $\varphi_{1}^{\prime \prime} \in C_{c}^{\infty}(N)$. Consequently

$$
\begin{aligned}
\kappa(\nu, \sigma)\left(f_{\varphi}\right)= & \int_{N \times N^{\prime}} \varphi_{1}^{\prime \prime}(n) \varphi_{2}\left(n^{\prime}\right) \chi\left(n^{\prime}\right) d n d n^{\prime} \\
& \times \int_{M \times A} e^{\left(\nu+\rho_{\theta}\right)(\log a)} \varphi_{1}^{\prime}(m a) \lambda\left(\sigma_{\infty}(m) v\right) d m d a .
\end{aligned}
$$

Let $W_{v}(m)=\lambda\left(\sigma_{\infty}(m) v\right), v \in H(\sigma)_{\infty}$. We need

Lemma 2.5. Fix $\varphi \in C_{c}^{\infty}(M)$ and let $\lambda$ be as in (2.1). Consider

$$
E_{\varphi}\left(\nu_{0}, \eta_{0}, \sigma\right)=\int_{M} \varphi(m) W_{v}(m) d m
$$

Then $E_{\varphi}\left(\nu_{0}, \eta_{0}, \sigma\right)$ is continuous in $\nu_{0}$. Moreover, given $v \in H(\sigma)_{\infty}$, there exists a $\varphi$ such that $E_{\varphi}\left(\nu_{0}, \eta_{0}, \sigma\right) \neq 0$. 
Proof. We only have to check $\nu_{0}$ with $\operatorname{Re}\left(\nu_{0}\left(H_{\alpha}\right)\right)=0$ for some $\alpha \in \theta$, since otherwise $W_{v}(m)$ is continuous as a function on $M \times S\left(\tilde{w}_{l, \theta}\right)$. Suppose $\nu_{0}=\lim _{n} \nu_{n}$ with $\operatorname{Re}\left(\nu_{n}\left(H_{\alpha}\right)\right)>0, \forall \alpha \in \theta$. Choose $\psi_{0} \in C_{c}^{\infty}(M)$ such that $\bar{\delta} v=f_{\psi_{0}}$. Here $f_{\psi_{0}}$ is defined as $f_{\varphi}$ before with $G, M, A, N, \nu, \sigma_{\infty}$ replaced by $M, M_{0}, A_{M}, U \cap M, v_{0}, \eta_{0}$, respectively. Now, let for every function $h$ on $M$, $L_{m} h\left(m^{\prime}\right)=h\left(m^{-1} m^{\prime}\right)$. Then $C=\left\{L_{m} \psi_{0} \mid m \in \operatorname{Supp} . \varphi\right\}$ is compact. For $\nu \in\left(\mathfrak{a}_{M}\right)_{\mathbf{C}}^{*}$ and $\psi \in C_{c}^{\infty}(M)$, let $T_{\nu}(\psi)=\lambda\left(\nu, \eta_{0}\right)\left(f_{\psi}\right)$. Then $\lim _{n} T_{\nu_{n}}(\psi)=T_{\nu_{0}}(\psi)$. Now using a standard lemma on distributions, given $\epsilon>0$, there exists a positive integer $N(\epsilon)$ such that

$$
\left|T_{\nu_{0}}(\psi)-T_{\nu_{n}}(\psi)\right|<\epsilon
$$

for all $n \geqslant N(\epsilon)$ and all $\psi \in C$. The lemma is now a consequence of (2.1) and the definition of $E_{\varphi}\left(\nu_{0}, \eta_{0}, \sigma\right)$.

Using Lemma 2.5 and (2.4.2), we now see that for every $\varphi$ as above and any $\nu \in\left(\mathfrak{a}_{\theta}\right)_{\mathbf{C}}^{*}$, the function $\kappa(\nu, \sigma)\left(f_{\varphi}\right)$ is continuous as a function of $\nu_{0}$ for all $\nu_{0}$ with $\operatorname{Re}\left(\nu_{0}\left(H_{\alpha}\right)\right) \geqslant 0, \forall \alpha \in \theta$. Moreover, given a pair $\left(\nu_{0}, \nu\right)$ as above, $\varphi$ can be chosen so that $\kappa(\nu, \sigma)\left(f_{\varphi}\right) \neq 0$. Consequently by Theorem 1.3 and (2.4.1), $C_{\sigma}\left(\nu, \nu_{0}, \eta_{0}\right)$ is continuous for all such $\nu_{0}$ (i.e. $\left.\operatorname{Re}\left(\nu_{0}\left(H_{\alpha}\right)\right) \geqslant 0, \forall \alpha \in \theta\right)$. The proposition is now a consequence of the equality $C_{\sigma}\left(\nu, \nu_{0}, \eta_{0}\right)=1$ for $\nu+\nu_{0} \in S\left(\tilde{w}_{l}\right)$.

Corollary. The functional $\kappa(\nu, \sigma)$ extends to an entire function of $\nu \in\left(a_{\theta}\right)_{\mathbf{C}}^{*}$ and for every $\nu$ defines a nonzero Whittaker functional for $V(\nu, \sigma, \theta)_{\infty}$.

We also state a useful corollary of the proof of Lemma 2.5.

Proposition 2.6. Fix $\nu \in \mathfrak{a}_{\mathbf{C}}^{*}$ and $\eta \in \hat{M}_{0}$. Choose $\varphi \in C_{c}^{\infty}(G)$ and set

$$
W_{\varphi}(g, \nu, \eta)=\lambda(\nu, \eta)\left(I(\nu, \eta)_{\infty}(g) f_{\varphi}\right) .
$$

Then as a function on $G \times \mathfrak{a}_{\mathbf{C}}^{*}$, the function $W_{\varphi}(g, \nu, \eta)$ is continuous.

Proof. To extend this to other Weyl chambers one has to only use the functional equation satisfied by $W_{f}(g, \nu, \eta)$. We remark that having proved Proposition 2.6 first, Lemma 2.5 would become trivial.

Remark. We can now remove the assumption that $\pi_{v}, v$ archimedean, is fine from Proposition 3.2, Theorems 4.1, 4.2, and 5.1 of [27] and use them in full generality of a quasi-split group. In fact by the Corollary to Proposition 2.4 one can conclude that for each $\nu \in\left(a_{\theta}\right)_{\mathrm{C}}^{*}$, there exists $f \in V\left(\nu, \pi_{\infty}, \theta\right)_{K}$ such that $\kappa\left(\nu, \pi_{\infty}\right)(f)=0\left(\kappa\left(\nu, \pi_{\infty}\right)(f)\right.$ is the local component at infinity of the Fourier coefficient $E_{\chi}\left(s ; \hat{\phi} ; e ; P_{\theta, \mathbf{A}}\right)$ of the corresponding Eisenstein series (page 343 of [27]) whose nonvanishing is crucial to a number of interesting arithmetic results [27]). The fact that there exists a $K$-finite function $f$ for which $\kappa\left(\nu, \pi_{\infty}\right)(f) \neq 0$ is essential since the theory of Eisenstein series has only been developed for such functions. One purpose of the present section is to provide the details of the results at infinity necessary for the previously mentioned results of [27]. 
3. Local coefficients as Artin factors. In this section we shall state and prove the main result of this paper. More precisely, we shall prove that certain arithmetic factors defined in [27] are in fact equal to the corresponding Artin factors defined by local reciprocity law by Langlands [21]. We shall start by recalling their definitions.

Let $\theta \subset \Delta$ and choose $\tilde{w} \in W$ such that $\tilde{w}(\theta) \subset \Delta$. Fix a representative $w \in G$ for $\tilde{w}$ and let $N_{\tilde{w}}=U \cap w N_{\theta}^{-} w^{-1}$, where $N_{\theta}^{-}$is the unipotent group generated by the roots in $\Sigma^{-}-\Sigma_{\theta}^{-}$. Let $\nu \in\left(\mathfrak{a}_{\theta}\right)_{\mathbf{C}}^{*}$ and fix an irreducible admissible $\chi$-generic Banach representation $(\sigma, H(\sigma))$ of $M_{\theta}^{0}=M$.

As in Section 2, fix $\nu_{0} \in\left(a_{M}\right)_{\mathrm{C}}^{*}$ and $\eta_{0} \in \hat{M}_{0}$ such that $\delta: H(\sigma)_{k} \subset V_{M}\left(\nu_{0}, \eta_{0}\right)_{K}$. Since $\sigma$ is generic, by Proposition 2.2 we can identify $H(\sigma)_{\infty}$ as a subspace of $V_{M}\left(\nu_{0}, \eta_{0}\right)_{\infty}$. Consequently $\tilde{\delta}=\operatorname{Ind} \bar{\delta}: V(\nu, \sigma, \theta)_{\infty} \subset V\left(\nu+\nu_{0}, \eta_{0}\right)_{\infty}$. Given $f \in$ $V(\nu, \sigma, \theta)_{\infty}$, let $\tilde{f}$ be its image in $V\left(\nu+\nu_{0}, \eta_{0}\right)_{\infty}$, and define

$$
A(\nu, \sigma, w) f=\tilde{\delta}^{-1} \cdot A\left(\nu+\nu_{0}, \eta_{0}, w\right) \tilde{f}
$$

where $A\left(\nu+\nu_{0}, \eta_{0}, w\right) \tilde{f}$ is defined by

$$
A\left(\nu+\nu_{0}, \eta_{0}, w\right) \tilde{f}(g)=\int_{N_{\tilde{w}}} \tilde{f}(g n w) d n \quad(g \in G) .
$$

This integral is absolutely convergent if for each reduced root $\alpha \in \Sigma_{\theta}^{+}$with $\tilde{w}(\alpha) \in \Sigma^{-}, \operatorname{Re}\left(\nu\left(H_{\alpha}\right)\right)$ is sufficiently large $[12,13,24]$. Here we have extended $\nu$ to $\mathfrak{a}_{0}$ by making it zero on $\mathfrak{a}_{M}=\mathfrak{a}_{M_{\theta}}$. Moreover for every such $\nu, A(\nu, \sigma, w) f$ $\in V(\tilde{w}(\nu), \tilde{w}(\sigma), \tilde{w}(\theta))_{\infty}$ and the map $A(\nu, \sigma, w)$ is continuous with respect to the corresponding Schwartz topologies. Also (using 3.2)), as a function of $\nu$, it extends to a meromorphic function of $\nu \in a_{\mathbf{C}}^{*}, \mathfrak{a}=a_{\theta}$ (cf. $\left.[12,13,24]\right)$. Observe that for $f \in V(\nu, \sigma, \theta)_{\infty}$,

$$
A(\nu, \sigma, w) f(g)=\int_{N_{\tilde{w}}} f(g n w) d n \quad(g \in G) .
$$

Now, let $\kappa(\nu, \sigma)$ and $\kappa(\tilde{w}(\nu), \tilde{w}(\sigma))$ be the corresponding Whittaker functionals for $V(\nu, \sigma, \theta)_{\infty}$ and $V(\tilde{w}(\nu), \tilde{w}(\sigma), \tilde{w}(\theta))_{\infty}$, defined by (2.2), respectively (we shall use analytic continuation of $\kappa(\nu, \sigma)$ to other chambers to define $\kappa(\tilde{w}(\nu), \tilde{w}(\sigma)))$. Then by Theorem 1.1 , for every $\nu$ with $\operatorname{Re}\left(\nu\left(H_{\alpha}\right)\right) \gg 0, \forall \alpha \in \Sigma_{\theta}^{+}$, there exists a complex number $C_{\chi}(\nu, \sigma, \theta, w)$, a priori may be identically infinity, such that (Theorem 3.1 of [27])

$$
\kappa(\nu, \sigma)(f)=C_{\chi}(\nu, \sigma, \theta, w) \kappa(\tilde{w}(\nu), \tilde{w}(\sigma))(A(\nu, \sigma, w)(f)),
$$

where $f \in V(\nu, \sigma, \theta)_{\infty}$. Consequently by corollary of Proposition $2.4, C_{\chi}(\nu, \sigma, \theta, w)$ extends to a meromorphic function of $\nu$ on $\mathfrak{a}_{\mathbf{C}}^{*}$. Moreover, an argument similar to the proof of Theorem 3.1 of [27] shows that for an open dense subset of $a_{\mathbf{C}}^{*}$, the function

$$
\kappa(\tilde{w}(\nu), \tilde{w}(\sigma)) A(\nu, \sigma, w)
$$


is not identically zero, and therefore, using (2.2) and (2.3), (3.3) implies that

$$
C_{\chi}(\nu, \sigma, \theta, w)=C_{\chi}\left(\nu_{0}+\nu, \eta_{0}, \varnothing, w\right) .
$$

Using Proposition 3.2.1 of [27] and Lemma 1.4 of the present paper, $C_{\chi}\left(\nu_{0}+\nu, \eta_{0}, \varnothing, w\right)$ can now be explicitly computed. The purpose of this section is to interpret these explicit formulas in terms of the Langlands' Weil group data attached to $\sigma$ by local reciprocity law at infinity [21].

To explain our result, let $F$ denote either $\mathbf{R}$ or $\mathbf{C}$. Let $W_{F}$ be the Weil group of $F$. It is equal to $\mathbf{C}^{*}$ if $F=\mathbf{C}$; and consists of pairs $(z, \tau), z \in \mathbf{C}^{*}$, $\tau \in \mathfrak{g}(\mathbf{C} / \mathbf{R})=\left\{1, \sigma_{0}\right\}$, the Galois group of $\mathbf{C}$ over $\mathbf{R}$, with multiplication

$$
\left(z_{1}, \tau_{1}\right)\left(z_{2}, \tau_{2}\right)=\left(z_{1} \tau_{1}\left(z_{2}\right) a_{\tau_{1}, \tau_{2}}, \tau_{1} \tau_{2}\right)
$$

where $a_{\tau_{1}, \tau_{2}}=1$ if $\tau_{1}=1$ or $\tau_{2}=1$ and $a_{\tau_{1}, \tau_{2}}=-1$ if $\tau_{1}=\tau_{2}=\sigma_{0}$, if $F=\mathbf{R}$.

When $\mathbf{G}$ is a complex group, we may, as we in fact do, by restriction of scalars consider $\mathbf{G}$ as a group over $\mathbf{R}$. For this reason from now on we shall assume $F=\mathbf{R}$.

Let $\varphi$ be the homomorphism of $W_{\mathbf{R}}$ into ${ }^{L} M$ the $L$-group of $\mathbf{M}$, attached to $\sigma$ (cf. [21]). Moreover, if ${ }^{L} N$ is the $L$-group of $\mathbf{N}=\mathbf{N}_{\theta}$, let ${ }^{L} \mathfrak{n}$ be its Lie algebra, and let $L_{\mathfrak{n}_{\tilde{w}}} \subset^{L} \mathfrak{n}$, be the subspace generated by those roots $\alpha^{\vee}$ for which $\tilde{w}(\alpha)$ is negative. Denote by $\rho$ the adjoint action of ${ }^{L} M$ on ${ }^{L} \mathfrak{n}_{\tilde{w}}$. Then $\rho \cdot \varphi$ is a complex representation of $W_{\mathbf{R}}$. Let $a_{1}, \ldots, a_{n}$ be the distinct values of $2 \rho_{\theta}\left(\alpha^{\vee}\right), \alpha \in \Sigma_{\theta}^{+}$ with $\tilde{w}(\alpha) \in \Sigma^{-}$, where $2 \rho_{\theta}$ is as before the sum of roots in $\Sigma_{\theta}^{+}$. Now, for each $i$, $i=1, \ldots, n$, let $V_{i} \subset^{L_{\mathfrak{n}_{\tilde{w}}}}$ be the subspace of ${ }^{L} \mathfrak{n}_{\tilde{w}}$ generated by those roots $\alpha^{\vee}$ for which $2 \rho_{\theta}\left(\alpha^{\vee}\right)=a_{i}$. Then each $V_{i}$ is $W_{\mathbf{R}^{-}}$-invariant under $\rho \cdot \varphi$ (as well as $\tilde{\rho} \cdot \varphi)$. Let $r_{i}=\rho \cdot \varphi \mid V_{i}$ and let $\tilde{r}_{i}$ be its contragradient.

Finally to specify $w$, fix a reduced decomposition of $\tilde{w}$ and for each simple reflection in the decomposition choose a representative as in Lemma 1.4. Now, let $w$ be their product according to this decomposition. We shall use this decomposition when we apply Proposition 3.2.1 of [27] to compute $C_{\chi}\left(\nu_{0}+\nu, \eta_{0}\right.$, $\varnothing, w)$.

We fix $\chi$ as follows. Let $\psi_{\mathbf{R}}(x)=\exp (2 \pi i x)$ and $\psi_{\mathbf{C}}(z)=\psi_{\mathbf{R}}\left(\operatorname{Tr}_{\mathbf{C} / \mathbf{R}}(z)\right.$ ) (as in Lemma 1.4). Now for every simple $\operatorname{root} \alpha$, let $\chi_{\alpha}\left(\exp \left(n_{\alpha} X_{\alpha}\right)\right)=\psi_{\mathbf{R}}\left(n_{\alpha}\right), n_{\alpha} \in \mathbf{R}$, or $\chi_{\alpha}\left(\exp \left(n_{\alpha} X_{\alpha}\right)\right)=\psi_{\mathbf{C}}\left(n_{\alpha}\right), n_{\alpha} \in \mathbf{C}$, according as $\alpha$ is real or complex. Here $X_{\alpha}$ is normalized by $\left[X_{\alpha}, X_{-\alpha}\right]=H_{\alpha}$, where $\alpha\left(H_{\alpha}\right)=2$.

Every complex irreducible representation of $W_{\mathbf{R}}$ is either one or two dimensional. In the latter case it is always of the form $\operatorname{Ind}_{W_{\mathbf{C}} \uparrow W_{\mathbf{R}}} \theta$, where $\theta$ is a character (not necessarily unitary) of $W_{\mathbf{C}}=\mathbf{C}^{*}$. Moreover, class field theory implies that a one-dimensional representation of $W_{\mathbf{R}}$ is in fact a character of $\mathbf{R}^{*}$. Fix a complex number $s$.

Assume first that $\tau$ is a one dimensional representation of $W_{\mathbf{R}}$. Write $\tau(x)=(x /|x|)^{\epsilon}|x|^{t}, t \in \mathbf{C}, \epsilon=0$ or 1 . Define

$$
L(s, \tau)=\pi^{-1 / 2(\epsilon+t+s)} \Gamma(1 / 2(\epsilon+t+s))
$$


and

$$
\epsilon\left(s, \tau, \psi_{\mathbf{R}}\right)=i^{\epsilon},
$$

i.e., the Hecke factors attached to $\tau$ as a character of $\mathbf{R}^{*}$.

Next, suppose $\tau$ is a two dimensional irreducible representation of $W_{\mathbf{R}}$. Then $\tau=\operatorname{Ind}_{\mathbf{C}^{*} \uparrow W_{\mathbf{R}}} \theta$, where $\theta(z)=|z|_{\mathbf{C}}^{t}(z / \bar{z})^{n / 2}, z \in \mathbf{C}^{*}, t \in \mathbf{C},|z| \mathbf{C}=z \bar{z}$, and $n \in \mathbf{Z}$. Define

$$
L(s, \tau)=2 \cdot(2 \pi)^{-(s+t+|n| / 2)} \Gamma(s+t+|n| / 2),
$$

the Hecke $L$-function attached to $\theta$. Moreover, let

$$
\epsilon_{\mathbf{C}}\left(s, \theta, \psi_{\mathbf{C} / \mathbf{R}}\right)=i^{|n|}
$$

be the corresponding root number, and as in [18], define

$$
\lambda\left(\mathbf{C} / \mathbf{R}, \psi_{\mathbf{R}}\right)=i .
$$

Then the root number attached to $\tau$ is given by (cf. [18])

$$
\epsilon\left(s, \tau, \psi_{\mathbf{R}}\right)=\lambda\left(\mathbf{C} / \mathbf{R}, \psi_{\mathbf{R}}\right) \epsilon_{\mathbf{C}}\left(s, \theta, \psi_{\mathbf{C} / \mathbf{R}}\right) .
$$

Now, if $\tau=\bigoplus_{j} \tau_{j}$ with irreducible $\tau_{j}$ 's; define

$$
L(s, \tau)=\prod_{j} L\left(s, \tau_{j}\right)
$$

and

$$
\epsilon\left(s, \tau, \psi_{\mathbf{R}}\right)=\prod_{j} \epsilon\left(s, \tau_{j}, \psi_{\mathbf{R}}\right)
$$

Finally, we set

$$
\gamma\left(s, \tau, \psi_{\mathbf{R}}\right)=\epsilon\left(s, \tau, \psi_{\mathbf{R}}\right) L(1-s, \tilde{\tau}) / L(s, \tau),
$$

where $\tilde{\tau}$ is the contragredient of $\tau$. Then the main result of this paper is the following:

THEOREM 3.1. Let $\sigma$ be an irreducible admissible $\chi$-generic Banach (in particular unitary $\chi$-generic) representation of $M$. Then

$$
C_{\chi}\left(-2 s \rho_{\theta}, \sigma, \theta, w\right)=\lambda\left(\mathbf{C} / \mathbf{R}, \psi_{\mathbf{R}}\right)^{2 m+p} \prod_{i=1}^{n} \epsilon\left(a_{i} s, r_{i}, \psi_{\mathbf{R}}\right) \frac{L\left(1-a_{i} s, \tilde{r}_{i}\right)}{L\left(a_{i} s, r_{i}\right)}
$$

where $m$ and $p$ are the number of rank one groups $G_{\alpha}, \alpha$ reduced, $\alpha \in \Sigma_{\theta}^{+}$, $\tilde{w}(\alpha) \in \Sigma^{-}$, whose simply connected coverings are isomorphic to $\mathrm{SU}(2,1)$ and $\mathrm{SL}(2, \mathbf{C})$, respectively. 
COROLlary. Let $\sigma$ be an irreducible admissible $\chi^{\prime}$-generic Banach representation of $M, \chi^{\prime} \neq \chi$. Then $\chi^{\prime}$ can be chosen in such a way that up to a determinable sign $C_{\chi^{\prime}}\left(-2 s \rho_{\theta}, \sigma, \theta, w\right)$ is equal to the right hand side of (3.1.1).

Remark 1. Theorem 3.1 and its corollary are the most general result that at the present time one can prove in the direction of decomposing the local coefficients in terms of representations $r_{i}$ of $W_{\mathbf{R}}$. In fact having the properties of Artin factors in hand, Theorem 3.1 allows us to treat the archimedean places the same as the nonarchimedean unramified ones (cf. [20]).

Remark 2. Let $m$ and $n$ be two positive integers and let $\mathbf{G}=\mathbf{G L}_{n+m}$. Fix $\mathbf{M}=\mathbf{G L}_{n} \times \mathbf{G L}_{m}$. Now Theorem 3.1 together with Theorem 5.1 of [28], when applied to Theorem 4.1 of [27] will give a new proof of the functional equation satisfied by the Rankin-Selberg $L$-functions attached to the pairs of cusp forms on $\mathbf{G L}_{n}\left(\mathbf{A}_{F}\right)$ and $\mathbf{G L}_{m}\left(\mathbf{A}_{F}\right)\left(\mathbf{A}_{F}\right.$ is the ring of adeles of a global field $\left.F\right)$ introduced by H. Jacquet, I. I. Piatetski-Shapiro, and J. A. Shalika (cf. [7, 8]).

Remark 3. Corollary 2 is a consequence of the fact that if $\sigma$ is $\chi^{\prime}$-generic, then $\chi^{\prime}$ can be chosen to be of the form $\chi^{\prime}=\prod \chi_{\alpha}^{\prime}, \alpha$ simple, with $\chi_{\alpha}^{\prime}(x)=\chi_{\alpha}\left(\epsilon_{\alpha} x\right)$, $\epsilon_{\alpha}= \pm 1$ (cf. [33]); combined with Proposition 3.2.1 of [27] applied to $C_{\chi^{\prime}}\left(\nu_{0}+\nu, \eta_{0}, \varnothing, w\right)$. It can also be stated as follows.

For each $i$, write $r_{i}=\bigoplus_{j} r_{i j}$, with $r_{i j}$ irreducible representations of $W_{\mathbf{R}}$. Then for each pair $(i, j)$, there exists a nontrivial additive character $\psi_{i j}$, determined by $\sigma$ and $\tilde{w}$, such that

$$
\begin{aligned}
C_{\chi^{\prime}}\left(-2 s \rho_{\theta}, \sigma, \theta, w\right) & =\lambda\left(\mathbf{C} / \mathbf{R}, \psi_{\mathbf{R}}\right)^{2 m+p} \cdot \prod_{i=1}^{n} \prod_{j} \epsilon\left(a_{i} s, r_{i j}, \psi_{i j}\right) \frac{L\left(1-a_{i} s, \tilde{r}_{i j}\right)}{L\left(a_{i} s, r_{i j}\right)} \\
& =\lambda\left(\mathbf{C} / \mathbf{R}, \psi_{\mathbf{R}}\right)^{2 m+p} \prod_{i=1}^{n} \frac{L\left(1-a_{i} s, \tilde{r}_{i}\right)}{L\left(a_{i} s, r_{i}\right)} \prod_{j} \epsilon\left(a_{i} s, r_{i j}, \psi_{i j}\right) .
\end{aligned}
$$

We first establish a simple necessary lemma.

Suppose for a moment that we are in the rank one situation and assume $\tilde{G}_{D} \cong \mathrm{SU}(2,1)$. Fix $\nu \in \mathfrak{a}_{\mathbf{C}}^{*}$ and $\eta \in \hat{M}_{0}$. As in Lemma 1.4 choose $n \in \mathbf{Z}$ such that

$$
\eta\left(\operatorname{diag}\left(e^{i \theta}, e^{-2 i \theta}, e^{i \theta}\right)\right)=e^{i n \theta}
$$

Fix $w, \psi_{\mathbf{R}}$, and the measure $d u$ as in Lemma 1.4. Finally if $\alpha$ is the unique reduced root, define $\nu_{\alpha}=\nu\left(H_{\alpha}\right)$, where $\alpha\left(H_{\alpha}\right)=2$. The character $\eta$ defines a unitary character of $\mathbf{C}^{1}$, complex numbers of absolute value one, which we extend to $\mathbf{C}$ in the obvious manner. We shall again denote this by $\eta$.

Let $\gamma_{2 \alpha}$ be the coroot of $2 \alpha$ at -1 . More precisely for any root $\delta$, let $\delta$ ve its dual root (or coroot), i.e., the map from $\mathrm{SL}_{2}(\mathbf{R})$ to the group. Then $\gamma_{\delta}=\delta^{\vee}(-1)$. In the case in hand $\gamma_{2 \alpha}=\operatorname{diag}(-1,1,-1)$. We now define a character $\tilde{\eta}$ of $\mathbf{R}^{*}$ by $\tilde{\eta}(x)=(x /|x|)^{\epsilon_{2 \alpha}}$ where $\epsilon_{2 \alpha}=0$ or 1 according as $n$ is odd or even. 
Next, let $\alpha$ and $\beta$ be the simple roots of $\mathrm{SL}_{3}$. Then the coroots $\alpha^{\vee}$ and $\beta^{\vee}$ are simple roots of $\mathrm{PGL}_{3}(\mathbf{C})={ }^{L} \tilde{G}_{D}^{0}$. In the formalism of $L$-groups they are so normalized that $\nu_{\alpha}=\nu\left(H_{\alpha}\right)=4 \nu\left(\alpha^{\vee}\right)$ for every $\nu \in \mathrm{a}_{\mathbf{C}}^{*}$. In fact fixing $\nu=\alpha+\beta$, $\nu\left(\alpha^{\vee}\right)=2\langle\alpha+\beta, \alpha\rangle /\langle\alpha, \alpha\rangle=1$, while on $\operatorname{SU}(2,1)$, this represents $2 \alpha$, leading to $\nu\left(H_{\alpha}\right)=2\langle 2 \alpha, \alpha\rangle /\langle\alpha, \alpha\rangle=4$. Observe that if $\rho=\alpha+\beta$ is half the sum of positive roots, $\rho\left(\alpha^{\vee}+\beta^{\vee}\right)=2$ and therefore

$$
(-1)^{\epsilon_{2 \alpha}}=-(-1)^{\rho\left(\alpha^{\vee}+\beta^{\vee}\right)} \eta\left(\gamma_{2 \alpha}\right) \text {. }
$$

Let $L_{\mathbf{C}}(s, \eta)$ and $\epsilon_{\mathbf{C}}\left(s, \eta, \psi_{\mathbf{C} / \mathbf{R}}\right)$ be the Hecke $L$-function and root number attached to $\eta$ as defined by the right hand sides of (3.7) and (3.8), respectively. Similarly define $L_{\mathbf{R}}(s, \tilde{\eta})$ and $\epsilon_{\mathbf{R}}\left(s, \tilde{\eta}, \psi_{\mathbf{R}}\right)$ using the right hand sides of (3.5) and (3.6), respectively. Finally set

$$
\gamma_{\mathbf{C}}\left(s, \eta, \psi_{\mathbf{C} / \mathbf{R}}\right)=\epsilon_{\mathbf{C}}\left(s, \eta, \psi_{\mathbf{C} / \mathbf{R}}\right) L_{\mathbf{C}}\left(1-s, \eta^{-1}\right) / L_{\mathbf{C}}(s, \eta)
$$

and

$$
\gamma_{\mathbf{R}}\left(s, \tilde{\eta}, \psi_{\mathbf{R}}\right)=\epsilon_{\mathbf{R}}\left(s, \tilde{\eta}, \psi_{\mathbf{R}}\right) L_{\mathbf{R}}(1-s, \tilde{\eta}) / L_{\mathbf{R}}(s, \tilde{\eta})
$$

We now prove:

Lemma 3.2. Suppose $\tilde{G}_{D} \cong \mathrm{SU}(2,1)$. Then

$$
C_{\chi}(\nu, \eta, \varnothing, w)=-\lambda\left(\mathbf{C} / \mathbf{R}, \psi_{\mathbf{R}}\right) \gamma_{\mathbf{C}}\left(\nu\left(\alpha^{\vee}\right), \eta, \psi_{\mathbf{C} / \mathbf{R}}\right) \gamma_{\mathbf{R}}\left(\nu\left(\alpha^{\vee}+\beta^{\vee}\right), \tilde{\eta}, \psi_{\mathbf{R}}\right) .
$$

Proof. For simplicity let $\nu=\nu_{\alpha}=\nu\left(H_{\alpha}\right)=4 \nu\left(\alpha^{\vee}\right)$. Suppose first that $n$ is even. Then $\epsilon_{2 \alpha}=1$, and the lemma is a consequence of Lemma 1.4, relation (3.9), and the identities

$$
\Gamma(\nu / 4+|n| / 2) \Gamma(1-\nu / 4-|n| / 2)=\pi / \sin (|n| \pi / 2+\pi \nu / 4)
$$

and

$$
\Gamma(1-\nu / 4) \Gamma(1 / 2+\nu / 4)=\pi^{1 / 2} / 2^{1-\nu / 2} \sin (\pi \nu / 4) \Gamma(\nu / 2) .
$$

The odd case can be proved similarly.

Remark. The sign appearing in (3.2.1) takes an important meaning when one considers $\mathrm{SU}(2,1)$ over a nonarchimedean field. In fact, using the calculations in [9], one is led to the sign of $E / F$ when $E$ is the quadratic extension of $F$ over which $\mathbf{G}$ splits (cf. [30]). By the sign of $E / F$ we shall mean +1 or -1 according as -1 is or is not a norm for $N_{E / F}$.

Finally, we remark that when $\tilde{G}_{D} \cong \mathrm{SL}_{2}(\mathbf{C})$, again the formalism of $L$-groups implies $\nu\left(H_{\alpha}\right)=2 \nu\left(\alpha^{\vee}\right)$ and consequently the corresponding $\gamma(\eta, \nu)$ of Lemma 1.4 equals

$$
\gamma(\eta, \nu)=\gamma_{\mathbf{C}}\left(\nu\left(\alpha^{\vee}\right), \eta, \psi_{\mathbf{C} / \mathbf{R}}\right)
$$


We now start proving Theorem 3.1. We shall first prove the theorem when $\sigma$ is in the discrete series. Consequently we assume that $P$ is cuspidal, i.e., $M$ admits discrete series.

Throughout this section, we shall extensively use certain results of Knapp and Zuckerman in [15]. Since the paper is already in print we may use certain notations or results without explicitly mentioning them.

Let $\mathfrak{m}_{0}$ be the Lie algebra of $M_{0}$. Then $\mathfrak{a}_{0} \oplus \mathfrak{m}_{0}$ is a Cartan subalgebra of $\mathrm{g}$, with $\mathfrak{a}_{0}=\mathfrak{a} \oplus \mathfrak{a}_{M}$. By assumption on $M$, let $\mathfrak{b}$ be a Cartan subalgebra of $\mathfrak{m}$, the Lie algebra of $M$, whose intersection with $m_{D e r}$ is compact. We fix a fundamental sequence $\tilde{\delta}_{1}, \ldots, \tilde{\delta}_{r}$ of positive noncompact $\left(\mathfrak{m}_{\mathbf{C}}, \mathfrak{b}_{\mathbf{C}}\right)$-roots. For each $\tilde{\delta_{j}}$, let $c_{\tilde{\delta}_{j}}$ be the corresponding Cayley transform (page 403 of [15]), and set $c=c_{\tilde{\delta}_{1}} \ldots c_{\tilde{\delta}_{r}}$. Then $\mathfrak{a}_{M}=c\left(\sum_{j=1}^{r} \mathbf{R} H_{\tilde{\delta}_{j}}\right)$. Let $\rho_{M}^{+}$be half the sum of positive $\left(\mathfrak{m}_{\mathbf{C}},\left(\mathfrak{a}_{M} \oplus \mathfrak{m}_{0}\right)_{\mathbf{C}}\right)-$ roots where the positive roots are chosen as in page 426 of [15].

The Cayley transform $c$ defines an isomorphism $c:(\mathfrak{a} \oplus \mathfrak{b})_{\mathbf{C}} \cong\left(\mathfrak{a}_{0} \oplus \mathfrak{m}_{0}\right)_{\mathbf{C}}$ by sending noncompact roots of $\left(\mathfrak{b} \ominus \mathrm{m}_{0}\right)_{\mathbf{C}}$ to the roots of $\left(\mathfrak{a}_{M}\right)_{\mathbf{C}}$. We shall use this isomorphism throughout the proof.

An $(\mathfrak{a} \oplus \mathfrak{b})_{\mathbf{C}}$-root $\epsilon$ is called real if $\epsilon \mid \mathfrak{b}=0$ and complex otherwise. If $\epsilon$ is an $\left(\mathfrak{a}_{0} \oplus \mathfrak{m}_{0}\right)_{\mathbf{C}}$-root, we call $\epsilon$ real if $\epsilon \mid \mathfrak{m}_{0}=0$. Otherwise it will again be called complex.

Let $\epsilon$ be a $\left(\mathfrak{g}_{\mathbf{C}},(\alpha \oplus \mathfrak{b})_{\mathbf{C}}\right)$-root. We call $\bar{\epsilon}$ its conjugate if $\langle\epsilon, H\rangle=\langle\bar{\epsilon}, H\rangle$ for $H \in \mathfrak{a}$, while $\langle\epsilon, H\rangle=-\langle\bar{\epsilon}, H\rangle$ for $H \in \mathfrak{b}$. We define the same notion for $\left(\mathfrak{g}_{\mathbf{C}},\left(\mathfrak{a}_{0} \oplus \mathfrak{m}_{0}\right)_{\mathbf{C}}\right)$-roots. The following simple lemma is useful.

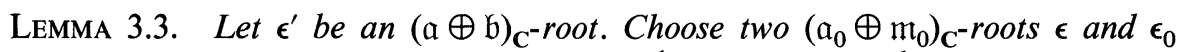
such that $\epsilon=c\left(\epsilon^{\prime}\right)$ and $\epsilon_{0}=c\left(\bar{\epsilon}^{\prime}\right)$. Let $\epsilon_{1}=c^{-1}(\bar{\epsilon})$ and $\epsilon_{2}=c^{-1}\left(\bar{\epsilon}_{0}\right)$. Then $\epsilon_{2}=\bar{\epsilon}_{1}$.

We shall now recall how $\varphi$ is defined.

Let $\mu$ be the Harish-Chandra parameter of $\sigma$. Denote by $B$ the Cartan subgroup of $M$ whose Lie algebra is $\mathfrak{b}$. Let $L=\operatorname{Hom}\left(\mathbf{C}^{*},{ }^{L} B^{0}\right)$ and $L^{\vee}$ $=\operatorname{Hom}\left({ }^{L} B^{0}, \mathbf{C}^{*}\right)$. If we choose $h=a \times\left(1 \times \sigma_{0}\right) \in{ }^{L} M$ as in Lemma 3.2 of [21], then with an appropriate choice of $a, \varphi\left(1 \times \sigma_{0}\right)=h$. Observe that $h \alpha^{\vee}=-\alpha^{\vee}$ for every root $\alpha^{\vee}$ of ${ }^{L} B^{0}$ in ${ }^{L} M^{0}$ (Lemma 3.2 of [21]). Let $\bar{\sigma}$ denote the action of $h$ on $L, L^{\vee}$, and ${ }^{L} B^{0}$. The homomorphism $\varphi$ is then defined so that (Lemma 3.4 of [21])

$$
\lambda^{\vee}(\varphi(z))=z^{\left\langle\mu, \lambda^{\vee}\right\rangle} \bar{z}_{\bar{z}}^{\left\langle\bar{\sigma} \mu, \lambda^{\vee}\right\rangle}
$$

for all $z \in \mathbf{C}^{*}=W_{\mathbf{C}}$ and $\lambda^{\vee} \in L^{\vee}$. Consequently, while $\rho \cdot \varphi\left(1 \times \sigma_{0}\right)$ fixes every real $(\mathfrak{a} \oplus \mathfrak{b})_{\mathbf{C}}$-root it permutes each pair of conjugate complex $(\mathfrak{a} \oplus \mathfrak{b})_{\mathbf{C}}$-roots, generating a two dimensional irreducible representation of $W_{\mathbf{R}}$.

We start with those $a_{0}$-roots $\alpha \in \Sigma_{\theta}^{+}$, with $\tilde{w}(\alpha) \in \Sigma^{-}$which are restrictions of complex $\left(a_{0} \oplus \mathfrak{m}_{0}\right)_{\mathbf{C}^{-}}$-roots. Their images under $c^{-1}$ are then restrictions of complex $(\mathfrak{a} \oplus \mathfrak{b})_{\mathbf{C}^{-}}$-roots. Let $\alpha^{\vee}: \mathbf{C}^{*} \rightarrow \mathrm{SL}_{2}(\mathbf{C})$ be the corresponding coroot and set $\tilde{\nu}=\nu+\nu_{0}$, where as before $\nu \in \mathfrak{a}_{\mathbf{C}}^{*}$ and $\nu_{0} \in\left(\mathfrak{a}_{M}\right)_{\mathrm{C}}^{*}$. We prove:

LeMma 3.4. Choose two complex $\left(\mathfrak{a}_{0} \oplus \mathfrak{m}_{0}\right)_{\mathbf{C}^{-}}$roots $\epsilon$ and $\epsilon_{0}$ such that $\epsilon \mid \mathfrak{a}_{0}=\alpha$ and $\epsilon_{0}=c\left(\overline{c^{-1}(\epsilon)}\right)$ (cf. Lemma 3.3). Let $\epsilon_{1}=c^{-1}(\bar{\epsilon})$ and denote by $\tau^{\prime}$ and $\tau_{1}$ the 
irreducible restrictions of $\rho \cdot \varphi$ to the two dimensional root spaces generated by $\left\{X_{\epsilon^{\prime} \vee}, X_{\bar{\epsilon}^{\prime}} \vee\right\}$ and $\left\{X_{\epsilon_{1}^{\vee}}, X_{\bar{\epsilon}_{1}^{\vee}}\right\}$, respectively. Then

$$
\begin{aligned}
& \lambda\left(\mathbf{C} / \mathbf{R}, \psi_{\mathbf{R}}\right)^{2} \gamma_{\mathbf{C}}\left(\tilde{\nu}\left(\alpha^{\vee}\right), \eta_{0} \cdot \alpha^{\vee}, \psi_{\mathbf{C} / \mathbf{R}}\right) \gamma_{\mathbf{C}}\left(\tilde{\nu}\left(\alpha_{0}^{\vee}\right), \eta_{0} \cdot \alpha_{0}^{\vee}, \psi_{\mathbf{C} / \mathbf{R}}\right) \\
& =\gamma\left(\nu\left(\epsilon^{\prime \vee}\right), \tau^{\prime}, \psi_{\mathbf{R}}\right) \gamma\left(\nu\left(\epsilon_{1}^{\vee}\right), \tau_{1}, \psi_{\mathbf{R}}\right) .
\end{aligned}
$$

Proof. Set $\tilde{\mu}=\mu+\nu$. Then by equivalence of infinitesimal characters (for example) for $\sigma$ and $I_{M}\left(\nu_{0}, \eta_{0}\right)$ one concludes that up to an element of $W\left(A_{M} M_{0}:(M A)^{\mathrm{C}}\right)$ which we may assume is equal to 1

$$
\tilde{\nu} \oplus \eta_{0}=c(\tilde{\mu}) .
$$

Applying (3.4.2) to $\epsilon, \epsilon_{0}$ and their conjugates, we obtain

$$
\begin{aligned}
& \tilde{\nu}\left(\alpha^{\vee}\right)=\left(\tilde{\mu}\left(\epsilon^{\prime \vee}\right)+\tilde{\mu}\left(\epsilon_{1}^{\vee}\right)\right) / 2, \\
& \tilde{\nu}\left(\alpha_{0}^{\vee}\right)=\left(\tilde{\mu}\left(\bar{\epsilon}^{\prime \vee}\right)+\tilde{\mu}\left(\bar{\epsilon}_{1}^{\vee}\right)\right) / 2, \\
& n\left(\eta_{0} \cdot \alpha^{\vee}\right)=\tilde{\mu}\left(\epsilon^{\prime \vee}\right)-\tilde{\mu}\left(\epsilon_{1}^{\vee}\right),
\end{aligned}
$$

and

$$
n\left(\eta_{0} \cdot \alpha_{0}^{\vee}\right)=\tilde{\mu}\left(\bar{\epsilon}^{\prime \vee}\right)-\tilde{\mu}\left(\bar{\epsilon}_{1}^{\vee}\right)
$$

where for every character $\lambda$ of $\mathbf{C}^{1}$, the integer $n(\lambda)$ is defined by

$$
\lambda\left(e^{i \theta}\right)=e^{i n(\lambda) \theta}
$$

Moreover $\tilde{\mu}\left(\epsilon^{\vee \vee}\right)-\tilde{\mu}\left(\bar{\epsilon}^{\prime \vee}\right)$ and $\tilde{\mu}\left(\epsilon_{1}^{\vee}\right)-\tilde{\mu}\left(\bar{\epsilon}_{1}^{\vee}\right)$ are also integers (cf. [21]). The following simple lemma is useful.

LEMMA 3.5. (a) Let $z$ and $z^{\prime}$ be two complex numbers such that $z-z^{\prime}$ is an integer. Set

$$
c\left(z, z^{\prime}\right)=i^{z-z^{\prime}} \cdot 2(2 \pi)^{-\left(1-z^{\prime}\right)} \Gamma\left(1-z^{\prime}\right) / 2(2 \pi)^{-z} \Gamma(z) .
$$

Then

$$
c\left(z, z^{\prime}\right)=i^{z-z^{\prime}} \cdot(2 \pi)^{z+z^{\prime}} / 2 \Gamma(z) \Gamma\left(z^{\prime}\right) \sin \left(\pi z^{\prime}\right) .
$$

In particular $c\left(z, z^{\prime}\right)=c\left(z^{\prime}, z\right)$.

(b) Let $z_{1}, z_{2}, z_{3}$, and $z_{4}$ be four complex numbers such that $z_{1}-z_{2}, z_{3}-z_{4}$, $z_{1}-z_{3}$, and $z_{2}-z_{4}$ are integers. Then

$$
c\left(z_{1}, z_{2}\right) c\left(z_{3}, z_{4}\right)=c\left(z_{1}, z_{3}\right) c\left(z_{2}, z_{4}\right) .
$$


Next observe that

$$
\begin{gathered}
\gamma_{\mathbf{C}}\left(\tilde{\nu}\left(\alpha^{\vee}\right), \eta_{0} \cdot \alpha^{\vee}, \psi_{\mathbf{C} / \mathbf{R}}\right)=c\left(\tilde{\mu}\left(\epsilon^{\vee \vee}\right), \tilde{\mu}\left(\epsilon_{1}^{\vee}\right)\right), \\
\gamma_{\mathbf{C}}\left(\tilde{\nu}\left(\alpha_{0}^{\vee}\right), \eta_{0} \cdot \alpha_{0}^{\vee}, \psi_{\mathbf{C} / \mathbf{R}}\right)=c\left(\tilde{\mu}\left(\bar{\epsilon}^{\vee \vee}\right), \tilde{\mu}\left(\bar{\epsilon}_{1}^{\vee}\right)\right), \\
\gamma\left(\nu\left(\epsilon^{\prime \vee}\right), \tau^{\prime}, \psi_{\mathbf{C} / \mathbf{R}}\right)=\lambda\left(\mathbf{C} / \mathbf{R}, \psi_{\mathbf{R}}\right) c\left(\tilde{\mu}\left(\epsilon^{\prime \vee}\right), \tilde{\mu}\left(\bar{\epsilon}^{\prime \vee}\right)\right),
\end{gathered}
$$

and

$$
\gamma\left(\nu\left(\epsilon_{1}^{\vee}\right), \tau_{1}, \psi_{\mathbf{C} / \mathbf{R}}\right)=\lambda\left(\mathbf{C} / \mathbf{R}, \psi_{\mathbf{R}}\right) c\left(\tilde{\mu}\left(\epsilon_{1}^{\vee}\right), \tilde{\mu}\left(\bar{\epsilon}_{1}^{\vee}\right)\right),
$$

using the discussion after Lemma 2.3 of [2]. Lemma 3.4 now follows if we apply part b) of Lemma 3.5 to $z_{1}=\tilde{\mu}\left(\epsilon^{\prime \vee}\right), z_{2}=\tilde{\mu}\left(\epsilon_{1}^{\vee}\right), z_{3}=\tilde{\mu}\left(\bar{\epsilon}^{\prime \vee}\right)$, and $z_{4}=\tilde{\mu}\left(\bar{\epsilon}_{1}^{\vee}\right)$.

Remark. Suppose $\epsilon_{1}=\epsilon^{\prime}$ or $\bar{\epsilon}^{\prime}$. Then $\epsilon=\epsilon_{0}$ or $\bar{\epsilon}_{0}$ and Lemma 3.4 trivially reduces to

$$
\lambda\left(\mathbf{C} / \mathbf{R}, \psi_{\mathbf{R}}\right) \gamma_{\mathbf{C}}\left(\tilde{\nu}\left(\alpha^{\vee}\right), \eta_{0} \cdot \alpha^{\vee}, \psi_{\mathbf{C} / \mathbf{R}}\right)=\gamma\left(\nu\left(\epsilon^{\prime \vee}\right), \tau^{\prime}, \psi_{\mathbf{R}}\right) .
$$

We shall now consider those $a_{0}$-roots $\alpha \in \Sigma_{\theta}^{+}$, with $\tilde{w}(\alpha) \in \Sigma^{-}$which are restrictions of real $\left(\mathfrak{a}_{0} \oplus \mathfrak{m}_{0}\right)_{\mathbf{C}}$-roots. With abuse of terminology we shall call these roots, real $a_{0}$-roots. For the moment we leave aside the case when $G$ has a factor of type $G_{2}$. Then, using Lemmas 7.4 and 7.5 of [15], we shall divide them to three categories.

The first category consists of those real $\mathfrak{a}_{0}$-roots $\beta$ which restrict to complex a-roots and are not of type $(\mathrm{dl})$ of Lemma 7.4 of [15]. More precisely, if $\epsilon^{\prime}$ is a real $\left(\mathfrak{a}_{0} \oplus \mathrm{m}_{0}\right)_{\mathbf{C}}$-root which restricts to $\beta$ (not of type $(\mathrm{d} 1)$ ), we would like that $c^{-1}\left(\epsilon^{\prime}\right)$ be a complex $(\mathfrak{a} \oplus \mathfrak{b})_{\mathbf{C}^{-r o o t}}$. Then by Lemma 7.6 of [15], neither $2 \beta$ nor $\beta / 2$ is an $a_{0}$-root. By Lemmas 7.4 and 7.5 of [15], there always exists an $\mathfrak{a}_{M}$-root $\delta$ which is the Cayley transform of a $\tilde{\delta_{j}}$ such that

$$
2\langle\beta, \delta\rangle /|\delta|^{2}= \pm 1
$$

and

$$
2\left\langle\rho_{M}^{+}, \delta\right\rangle /|\delta|^{2} \equiv 1(\bmod 2) .
$$

Following Knapp-Zuckerman (page 433 of [15]), we shall now group $\beta$ with three other such real $\mathfrak{a}_{0}$-roots. The procedure is entirely due to them. We recall this procedure.

Let

$$
p_{\delta} \beta=\beta-r \delta
$$

be the projection of $\beta$ along $\delta, r=2\langle\beta, \delta\rangle /|\delta|^{2}$. Then for such roots $r= \pm|\beta|^{2} /|\delta|^{2}$. For simplicity, let $\beta_{0}=p_{\delta} \beta$. Let $\alpha=\beta \mid a$ be the corresponding 
a-root. Then $\beta=\alpha+\alpha_{I}$ with $\alpha_{I} \neq 0$ a linear combination of $\mathfrak{a}_{M}$-roots. Let $\bar{\beta}=\alpha-\alpha_{I}$. Then $(\bar{\beta})_{0}=\overline{\beta_{0}}$. Also observe that since $\beta$ is real so is $\beta_{0}$ and by (3.19) we have

$$
\begin{aligned}
\gamma_{\beta_{0}} & =\gamma_{\beta} \cdot \gamma_{\delta}^{2\langle\beta, \delta\rangle /|\delta|^{2}} \\
& =\gamma_{\beta} \gamma_{\delta} .
\end{aligned}
$$

As before, for every real $a_{0}$-root $\delta^{\prime}$ define $\epsilon_{\delta^{\prime}}=0$ or 1 by

$$
\eta_{0}\left(\gamma_{\delta^{\prime}}\right)=(-1)^{\epsilon_{\delta^{\prime}}}
$$

Then by Lemma 7.2 of [15] and relation (3.20) above

$$
\begin{aligned}
\eta_{0}\left(\gamma_{\delta}\right) & =(-1)^{2\left\langle\nu_{0}-\rho_{M}^{+}, \delta\right\rangle /|\delta|^{2}} \\
& =-(-1)^{2\left\langle\nu_{0}, \delta\right\rangle /|\delta|^{2}} .
\end{aligned}
$$

Consequently (3.21) implies

$$
\epsilon_{\beta}+\epsilon_{\beta_{0}} \equiv 1+\frac{2\left\langle\nu_{0}, \delta\right\rangle}{|\delta|^{2}}(\bmod 2) .
$$

Similarly

$$
\epsilon_{\bar{\beta}}+\epsilon_{\bar{\beta}_{0}} \equiv 1+\frac{2\left\langle\nu_{0}, \delta\right\rangle}{|\delta|^{2}}(\bmod 2) .
$$

Let $\epsilon$ and $\epsilon_{0}$ be complex $(a \oplus \mathfrak{b})_{\mathbf{C}}$-roots such that $c(\epsilon)$ and $c\left(\epsilon_{0}\right)$ restrict to $\beta$ and $\beta_{0}$. Then $c(\bar{\epsilon})$ and $c\left(\bar{\epsilon}_{0}\right)$ restrict to $\bar{\beta}$ and $\bar{\beta}_{0}$, and if $\tilde{\mu}=\mu+\nu$, then by Lemma 7.2 of [15] and equality $\left|\beta_{0}\right|=|\beta|$

$$
\begin{aligned}
\tilde{\mu}\left(\epsilon_{0}^{\vee}\right) & =\frac{2\left\langle\nu+\nu_{0}, \beta_{0}\right\rangle}{\left|\beta_{0}\right|^{2}} \\
& =\tilde{\mu}\left(\epsilon^{\vee}\right)-r \cdot \frac{2\left\langle\nu_{0}, \delta\right\rangle}{|\delta|^{2}} \\
& =\tilde{\mu}\left(\epsilon^{\vee}\right) \pm \frac{2\left\langle\nu_{0}, \delta\right\rangle}{|\delta|^{2}} .
\end{aligned}
$$

Similarly

$$
\begin{aligned}
\tilde{\mu}\left(\bar{\epsilon}_{0}^{\vee}\right) & =\tilde{\nu}\left(\bar{\beta}_{0}^{\vee}\right) \\
& =\tilde{\mu}\left(\bar{\epsilon}^{\vee}\right) \mp \frac{2\left\langle\nu_{0}, \delta\right\rangle}{|\delta|^{2}} .
\end{aligned}
$$


We now prove

LEMMA 3.6. Let $\tau$ and $\tau_{0}$ be the irreducible restrictions of $\rho \cdot \varphi$ to the two dimensional root spaces generated by $\left\{X_{\epsilon^{\vee}}, X_{\bar{\epsilon}^{\vee}}\right\}$ and $\left\{X_{\epsilon_{0}^{\vee}}, X_{\bar{\epsilon}_{0}^{\vee}}\right\}$, respectively. Then

$$
\begin{aligned}
\gamma_{\mathbf{R}}\left(\tilde{\nu}\left(\beta^{\vee}\right), \eta_{0} \cdot \beta^{\vee}, \psi_{\mathbf{R}}\right) \gamma_{\mathbf{R}}\left(\tilde{\nu}\left(\beta_{0}^{\vee}\right), \eta_{0} \cdot \beta_{0}^{\vee}, \psi_{\mathbf{R}}\right) \\
\cdot \gamma_{\mathbf{R}}\left(\tilde{\nu}\left(\bar{\beta}^{\vee}\right), \eta_{0} \cdot \bar{\beta}^{\vee}, \psi_{\mathbf{R}}\right) \gamma_{\mathbf{R}}\left(\tilde{\nu}\left(\bar{\beta}_{0}^{\vee}\right), \eta_{0} \cdot \bar{\beta}_{0}^{\vee}, \psi_{\mathbf{R}}\right) \\
=\gamma\left(\nu\left(\epsilon^{\vee}\right), \tau, \psi_{\mathbf{R}}\right) \gamma\left(\nu\left(\epsilon_{0}^{\vee}\right), \tau_{0}, \psi_{\mathbf{R}}\right) .
\end{aligned}
$$

Proof. We start with a simple useful lemma.

Lemma 3.7. Suppose $\epsilon=0$ or 1 and fix a complex number $s$. Define a character $\eta_{\epsilon}$ of $\mathbf{R}^{*}$ by $\eta_{\epsilon}(x)=(x /|x|)^{\epsilon}$. Then

$$
\gamma_{\mathbf{R}}\left(s, \eta_{\epsilon}, \psi_{\mathbf{R}}\right)=i^{\epsilon} / 2(2 \pi)^{-s} \Gamma(s) \cos (\pi(s-\epsilon) / 2) .
$$

To continue, assume first that

$$
2\left\langle\nu_{0}, \delta\right\rangle /|\delta|^{2} \equiv 1(\bmod 2) .
$$

Then relations (3.23) and (3.24) imply that $\epsilon_{\beta}=\epsilon_{\beta_{0}}$ and $\epsilon_{\bar{\beta}}=\epsilon_{\bar{\beta}_{0}}$. Applying Lemma 3.7 to the factors in the left hand side of (3.6.1) implies that it is equal to the product of

$$
(2 \pi)^{\left(\tilde{\mu}\left(\epsilon^{\vee}\right)+\tilde{\mu}\left(\epsilon_{0}^{\vee}\right)+\tilde{\mu}\left(\bar{\epsilon}^{\vee}\right)+\tilde{\mu}\left(\tilde{\epsilon}_{0}^{\vee}\right)\right)}
$$

with

$$
\left(\Gamma\left(\tilde{\mu}\left(\epsilon^{\vee}\right)\right) \Gamma\left(\tilde{\mu}\left(\epsilon_{0}^{\vee}\right)\right) \Gamma\left(\tilde{\mu}\left(\bar{\epsilon}^{\vee}\right)\right) \Gamma\left(\tilde{\mu}\left(\bar{\epsilon}_{0}\right)\right)\right)^{-1}
$$

and

$$
(-1)^{\epsilon_{\beta}+\epsilon_{\bar{\beta}}}\left(-4 \sin \pi\left(\tilde{\mu}\left(\epsilon^{\vee}\right)-\epsilon_{\beta}\right) \sin \pi\left(\tilde{\mu}\left(\bar{\epsilon}^{\vee}\right)-\epsilon_{\bar{\beta}}\right)\right)^{-1},
$$

where we have repeatedly used (3.25), (3.26), and (3.6.2).

Checking all the possibilities for $\epsilon_{\beta}$ and $\epsilon_{\bar{\beta}}$, it is clear that (3.6.5) is always equal to

$$
-\left(4 \sin \pi \tilde{\mu}\left(\epsilon^{\vee}\right) \sin \pi \tilde{\mu}\left(\bar{\epsilon}^{\vee}\right)\right)^{-1} \text {. }
$$

On the other hand by (3.5.2) the right hand side of (3.6.1) is equal to the product of (3.6.3), (3.6.4),

$$
\lambda\left(\mathbf{C} / \mathbf{R}, \psi_{\mathbf{R}}\right)^{2} i^{\tilde{\mu}\left(\bar{\epsilon}^{\vee}\right)-\tilde{\mu}\left(\epsilon^{\vee}\right)+\tilde{\mu}\left(\epsilon_{0}^{\vee}\right)-\tilde{\mu}\left(\bar{\epsilon}_{0}^{\vee}\right)},
$$


and

$$
\left(4 \sin \pi \tilde{\mu}\left(\epsilon^{\vee}\right) \sin \pi \tilde{\mu}\left(\bar{\epsilon}_{0}^{\vee}\right)\right)^{-1} .
$$

But now by (3.25) and (3.26)

$$
\tilde{\mu}\left(\bar{\epsilon}^{\vee}\right)-\tilde{\mu}\left(\epsilon^{\vee}\right)+\tilde{\mu}\left(\epsilon_{0}^{\vee}\right)-\tilde{\mu}\left(\bar{\epsilon}_{0}^{\vee}\right)=2\left( \pm 2\left\langle\nu_{0}, \delta\right\rangle /|\delta|^{2}\right),
$$

while

$$
\sin \pi \tilde{\mu}\left(\bar{\epsilon}_{0}^{\vee}\right)=-\sin \pi \tilde{\mu}\left(\bar{\epsilon}^{\vee}\right),
$$

and the lemma in the odd case follows if one notices that (3.6.7) is equal to 1 , using (3.6.2) and (3.9).

The even case can be proved similarly, completing the lemma.

Next we shall consider all those real $\mathfrak{a}_{0}$-roots which are of type (d1) of Lemma 7.4 of [15]. They clearly restrict to complex a-roots. The following lemma explains a crucial fact about such roots. Its proof can be extracted from the discussions in page 436 of [15].

Lemma 3.8 (Knapp-Zuckerman). Let $\delta_{1}, \ldots, \delta_{C}$ be so that $\left\{\alpha \pm \frac{1}{2} \delta_{j} \mid 1 \leqslant j\right.$ $\leqslant C$ \} provides all the odd roots $\beta$ of type $(\mathrm{dl})$ restricting to $\alpha$, where $\delta_{j}$ 's are ordered according to their original ordering. Then

$$
2\left\langle\rho_{M}^{+}, \delta_{j}\right\rangle /\left|\delta_{j}\right|^{2} \equiv C+1-j(\bmod 2), \quad 1 \leqslant j \leqslant C .
$$

Suppose $C$ is odd. Group $\beta_{1}=\alpha+\frac{1}{2} \delta_{1}, \bar{\beta}_{1}=\alpha-\frac{1}{2} \delta_{1}$, and $2 \alpha$ together. We shall first consider other $\beta_{j}=\alpha+\frac{1}{2} \delta_{j}$, i.e., we assume $j \neq 1$. We then group them as $\left\{\beta_{j}, \bar{\beta}_{j}, \beta_{j+1}, \bar{\beta}_{j+1}\right\}$ with $1<j \leqslant C$. We now prove

Lemma 3.9. Suppose $C$ is odd. For each $1<j \leqslant C$, choose real $\left(\mathfrak{a}_{0} \oplus \mathfrak{m}_{0}\right)_{\mathrm{C}^{-}}$ roots $\epsilon_{j}^{\prime}$ and $\epsilon_{j+1}^{\prime}$ such that $\epsilon_{j}^{\prime} \mid a_{0}=\beta_{j}$ and $\epsilon_{j+1}^{\prime} \mid a_{0}=\beta_{j+1}$. Set $\epsilon_{j}=c^{-1}\left(\epsilon_{j}^{\prime}\right)$ and $\epsilon_{j+1}=c^{-1}\left(\epsilon_{j+1}^{\prime}\right)$. Let $\tau_{j}$ and $\tau_{j+1}$ be the irreducible restrictions of $\rho \cdot \varphi$ to the two dimensional root spaces generated by $\left\{X_{\epsilon_{v}^{\vee}}, X_{\overline{\bar{\epsilon}}_{j}}\right\}$ and $\left\{X_{\bar{\epsilon}_{j+1}^{\vee}}, X_{\overline{\bar{j}}_{j+1}}\right\}$. Let $S=\{2 k \mid 1 \leqslant k \leqslant(C-1) / 2\}$. Then for each $j \in S$

$$
\begin{aligned}
\gamma_{\mathbf{R}}\left(\tilde{\nu}\left(\beta_{j}^{\vee}\right), \eta_{0} \cdot \beta_{j}^{\vee}, \psi_{\mathbf{R}}\right) \gamma_{\mathbf{R}}\left(\tilde{\nu}\left(\bar{\beta}_{j}^{\vee}\right), \eta_{0} \cdot \bar{\beta}_{j}^{\vee}, \psi_{\mathbf{R}}\right) \\
\quad \cdot \gamma_{\mathbf{R}}\left(\tilde{\nu}\left(\beta_{j+1}^{\vee}\right), \eta_{0} \cdot \beta_{j+1}^{\vee}, \psi_{\mathbf{R}}\right) \gamma_{\mathbf{R}}\left(\tilde{\nu}\left(\bar{\beta}_{j+1}^{\vee}\right), \nu_{0} \cdot \bar{\beta}_{j+1}^{\vee}, \psi_{\mathbf{R}}\right) \\
=\gamma\left(\nu\left(\epsilon_{j}^{\vee}\right), \tau_{j}, \psi_{\mathbf{R}}\right) \gamma\left(\nu\left(\epsilon_{j+1}^{\vee}\right), \tau_{j+1}, \psi_{\mathbf{R}}\right) .
\end{aligned}
$$


Proof. Observe first that

$$
\begin{aligned}
\tilde{\nu}\left(\beta_{j}\right) & =2\left\langle\nu+\nu_{0}, \alpha+\frac{1}{2} \delta_{j}\right\rangle /\left|\beta_{j}\right|^{2} \\
& =\nu\left(\beta_{j}^{\vee}\right)+2\left\langle\nu_{0}, \delta_{j}\right\rangle / 2\left|\beta_{j}\right|^{2} \\
& =\nu\left(\beta_{j}^{\vee}\right)+2\left\langle\nu_{0}, \delta_{j}\right\rangle /\left|\delta_{j}\right|^{2} .
\end{aligned}
$$

Moreover $\gamma_{\beta_{j}}=\gamma_{2 \alpha} \gamma_{\delta_{j}}$ together with Lemma 7.2 of [15], imply that

$$
\epsilon_{\beta_{j}}=\epsilon_{\bar{\beta}_{j}} \equiv \epsilon_{2 \alpha}+\frac{2\left\langle\nu_{0}-\rho_{M}^{+}, \delta_{j}\right\rangle}{\left|\delta_{j}\right|^{2}}(\bmod 2) .
$$

Set $\nu_{\beta_{j}}=2\left\langle\nu_{0}, \delta_{j}\right\rangle /\left|\delta_{j}\right|^{2}$. Then by Lemma 3.8

$$
\epsilon_{\beta_{j}}=\epsilon_{\bar{\beta}_{j}} \equiv \epsilon_{2 \alpha}+\nu_{\beta_{j}}+C+1-j(\bmod 2) .
$$

Similarly for $j+1$. Observe that $\nu_{\bar{\beta}_{l}}=-\nu_{\beta_{l}}, l=j$ and $j+1$.

Using Lemmas 3.5 and 3.7, and the equalities $\tilde{\nu}\left(\beta_{l}^{\vee}\right)=\tilde{\mu}\left(\epsilon_{l}^{\vee}\right)$ and $\tilde{\nu}\left(\bar{\beta}_{l}^{\vee}\right)$ $=\tilde{\mu}\left(\bar{\epsilon}_{l}^{\vee}\right), l=j$ and $j+1$, proof of (3.9.1) is equivalent to equality of

$$
\begin{gathered}
i^{2 \epsilon_{\beta_{j}}+2 \epsilon_{\beta_{j+1}}}\left(4 \cos \frac{\pi}{2}\left(\tilde{\nu}\left(\beta_{j}^{\vee}\right)-\epsilon_{\beta_{j}}\right) \cos \frac{\pi}{2}\left(\tilde{\nu}\left(\bar{\beta}_{j}^{\vee}\right)-\epsilon_{\bar{\beta}_{j}}\right)\right. \\
\left.\cdot \cos \frac{\pi}{2}\left(\tilde{\nu}\left(\beta_{j+1}^{\vee}\right)-\epsilon_{\beta_{j+1}}\right) \cos \frac{\pi}{2}\left(\tilde{\nu}\left(\bar{\beta}_{j+1}^{\vee}\right)-\epsilon_{\bar{\beta}_{j+1}}\right)\right)^{-1}
\end{gathered}
$$

with

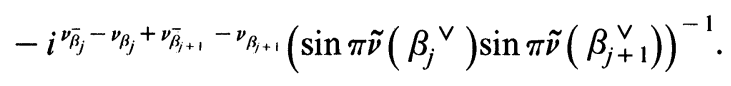

To prove this, let $c_{l}=\tilde{\nu}\left(\beta_{l}^{\vee}\right)-\epsilon_{\beta_{l}}, l=j, j+1$. Then

$$
\tilde{\nu}\left(\bar{\beta}_{l}^{\vee}\right)-\epsilon_{\bar{\beta}_{l}}=c_{l}-2 v_{\beta_{l}},
$$

$l=j, j+1$. Consequently

$$
\cos \frac{\pi}{2}\left(\tilde{\nu}\left(\bar{\beta}_{l}^{\vee}\right)-\epsilon_{\bar{\beta}_{l}}\right)=(-1)^{\nu_{\beta_{l}}} \cos \left(\pi c_{l} / 2\right) .
$$

$l=j, j+1$, and therefore (3.9.3) is equal to

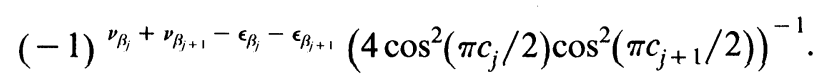

Moreover by (3.9.2)

$$
c_{j+1} \equiv c_{j}+1(\bmod 2)
$$


which immediately shows that (3.9.5) is equal to

$$
(-1)^{\nu_{\beta_{j}}+\nu_{\beta_{j+1}}-\epsilon_{\beta_{j}}-\epsilon_{\beta_{j+1}}}\left(\sin ^{2} \pi \tilde{\nu}\left(\beta_{j}^{\vee}\right)\right)^{-1} .
$$

Now (3.9.4) equals to

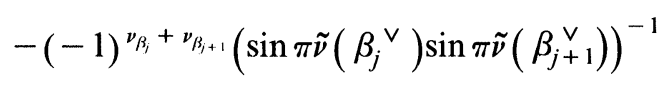

and to complete the lemma we only have to show

$$
\sin \pi \tilde{\nu}\left(\beta_{j+1}^{\vee}\right)=-(-1)^{\epsilon_{\beta_{j}}}+\epsilon_{\beta_{j+1}} \sin \pi \tilde{\nu}\left(\beta_{j}^{\vee}\right) .
$$

But this is a simple consequence of (3.9.6).

Remark. When $C$ is even this is all we have to do.

To complete the second category we now consider $\left\{\beta_{1}, \bar{\beta}_{1}, 2 \alpha=\beta_{1}+\bar{\beta}_{1}\right\}$. While $\alpha$ is not an $a_{0}$-root (Proposition 7 of [10]), $2 \alpha$ is one and restricts to a real a-root. Since $\alpha$ is not an $\mathfrak{a}_{0}$-root, $2 \alpha$ is a real reduced $a_{0}$-root; $\left(\tilde{G}_{2 \alpha}\right)_{D} \cong \operatorname{SL}_{2}(\mathbf{R})$ and the corresponding local coefficient is equal to $\gamma_{\mathbf{R}}\left(\tilde{\nu}\left((2 \alpha)^{\vee}\right), \eta_{0} \cdot(2 \alpha)^{\vee}, \psi_{\mathbf{R}}\right)$. For simplicity, let $\beta=\beta_{1}$ and $\delta=\delta_{1}$. We need the following lemma.

Lemma 3.10. Choose two real $\left(a_{0} \oplus \mathfrak{m}_{0}\right)_{\mathbf{C}^{-}}$roots $\epsilon^{\prime}$ and $\epsilon_{0}^{\prime}$ such that $\epsilon^{\prime} \mid \mathfrak{a}_{0}=\beta$ and $\epsilon_{0}^{\prime} \mid a_{0}=2 \alpha$. Set $\epsilon=c^{-1}\left(\epsilon^{\prime}\right)$ and $\epsilon_{0}=c^{-1}\left(\epsilon_{0}^{\prime}\right)$. Let $\tau$ and $\tau_{0}$ be the irreducible restrictions of $\rho \cdot \varphi$ to the two and one dimensional root spaces generated by $\left\{X_{\epsilon^{\vee}}, X_{\bar{\epsilon}^{\vee}}\right\}$ and $X_{\epsilon_{0}^{\vee}}$, respectively. Then

$$
\begin{aligned}
\gamma_{\mathbf{R}}\left(\tilde{\nu}\left(\beta^{\vee}\right), \eta_{0} \cdot \beta^{\vee}, \psi_{\mathbf{R}}\right) \gamma_{\mathbf{R}}\left(\tilde{\nu}\left(\bar{\beta}^{\vee}\right), \eta_{0} \cdot \bar{\beta}^{\vee}, \psi_{\mathbf{R}}\right) \\
\cdot \gamma \mathbf{R}\left(\tilde{\nu}\left((2 \alpha)^{\vee}\right), \eta_{0} \cdot(2 \alpha)^{\vee}, \psi_{\mathbf{R}}\right) \\
=\gamma\left(\nu\left(\epsilon^{\vee}\right), \tau, \psi_{\mathbf{R}}\right) \gamma\left(\nu\left(\epsilon_{0}^{\vee}\right), \tau_{0}, \psi_{\mathbf{R}}\right) .
\end{aligned}
$$

Proof. We define $\epsilon_{\beta}=\epsilon_{\bar{\beta}}, \epsilon_{2 \alpha}$, and $\nu_{\beta}=-\nu_{\bar{\beta}}$ as in Lemma 3.9. Moreover let $\nu_{2 \alpha}=2\langle\nu, 2 \alpha\rangle /|2 \alpha|^{2}$ which using $|2 \alpha|^{2}=2|\beta|^{2}$ is equal to $\tilde{\nu}\left(\beta^{\vee}\right)$. Since $C$ is odd, relation (7.25) of [15] implies that

$$
\begin{aligned}
\frac{2\left\langle\rho_{\alpha}, 2 \alpha\right\rangle}{|\alpha|^{2}} & \equiv C+1(\bmod 2) \\
& \equiv 0(\bmod 2)
\end{aligned}
$$

where $\rho_{\alpha}$ is half of the sum of positive $a_{0}$-roots restricting to $\alpha$ and $2 \alpha$. The element $\gamma_{2 \alpha}$ is central and therefore Lemma 7.3 of [15] together with Lemma 2.3 of [2] imply that $\rho \cdot \varphi(1 \times \sigma)$ acts on $X_{\epsilon_{0}^{\vee}}$ by $(-1)^{1-\epsilon_{2 \alpha}}$. Consequently by Lemma 
3.7 and the discussion after Lemma 2.3 of [2]

$$
\gamma\left(\nu\left(\epsilon_{0}^{\vee}\right), \tau_{0}, \psi_{\mathbf{R}}\right)=i^{1-\epsilon_{2 \alpha}}\left(2(2 \pi)^{-\nu_{2 \alpha}} \Gamma\left(\nu_{2 \alpha}\right) \cos \left(\pi\left(\nu_{2 \alpha}-1+\epsilon_{2 \alpha}\right) / 2\right)\right)^{-1} .
$$

Using an argument similar to the one given in Lemma 3.6, we only need to prove the equality of

$$
\begin{aligned}
& i^{\epsilon_{\beta}+\epsilon_{\bar{\beta}}+\epsilon_{2 \alpha}}\left(2 \cos \left(\pi\left(\tilde{\nu}\left(\beta^{\vee}\right)-\epsilon_{\beta}\right) / 2\right) \cos \left(\pi\left(\tilde{\nu}\left(\bar{\beta}^{\vee}\right)-\epsilon_{\bar{\beta}}\right) / 2\right)\right. \\
& \left.\cdot \cos \left(\pi\left(\nu_{2 \alpha}-\epsilon_{2 \alpha}\right) / 2\right)\right)^{-1}
\end{aligned}
$$

with

$$
-i^{\nu_{\bar{\beta}}-\nu_{\beta}-\epsilon_{2 \alpha}}\left(\sin \pi \tilde{\nu}\left(\beta^{\vee}\right) \cos \left(\pi\left(\nu_{2 \alpha}-1+\epsilon_{2 \alpha}\right) / 2\right)\right)^{-1} .
$$

We first prove the equality of root numbers, i.e.,

$$
i^{\epsilon_{\beta}+\epsilon_{\bar{\beta}}+\epsilon_{2 \alpha}}=-i^{\nu_{\bar{\beta}}-\nu_{\beta}-\epsilon_{2 \alpha}} .
$$

Since $2\left\langle\rho_{M}^{+}, \delta\right\rangle /|\delta|^{2}=C$ is odd, the equality $\gamma_{\bar{\beta}}=\gamma_{\beta}=\gamma_{2 \alpha} \gamma_{\delta}$ immediately implies

$$
\epsilon_{\beta} \equiv \epsilon_{2 \alpha}+\nu_{\beta}+1(\bmod 2)
$$

Now the left hand side of $(3.10 .4)$ is equal to

$$
(-1)^{\epsilon_{2 \alpha}+\nu_{\beta}+1} \cdot i^{\epsilon_{2 \alpha}}
$$

while the right hand side equals

$$
-(-1)^{\nu_{\beta}} \cdot(-i)^{\epsilon_{2 \alpha}}=(3.10 .6),
$$

proving the validity of (3.10.4).

The equality of the remaining terms in (3.10.2) and (3.10.3) can now be proved by letting $\nu_{\beta}$ be odd or even and checking the equality for all the possibilities of $\epsilon_{\beta}$ and $\epsilon_{2 \alpha}$ which are related by relation (3.10.5). The lemma is now complete.

It remains to check those real $\mathfrak{a}_{0}$-roots which restrict to real $\mathfrak{a}$-roots. In view of Lemma 3.10, there are only two possibilities.

LEMMA 3.11. Let $\alpha$ be a reduced real $\mathfrak{a}_{0}$-root which restricts to a real $\mathfrak{a}$-root. Moreover assume that if $\alpha / 2$ restricts to an a-root, then the number of pairs of odd $a_{0}$-roots of type $(\mathrm{d} 1)$ restricting to $\alpha / 2$, is even. Choose a real $\left(a_{0} \oplus \mathrm{m}_{0}\right)_{\mathbf{C}^{-r o o t}} \epsilon^{\prime}$ such that $\epsilon^{\prime} \mid \mathfrak{a}_{0}=\alpha$. Let $\epsilon=c^{-1}\left(\epsilon^{\prime}\right)$, and denote by $\tau$ the one dimensional restriction of $\rho \cdot \varphi$ to the root space generated by $X_{\epsilon^{\vee}}$. Then the corresponding local coefficient is equal to $\gamma_{\mathbf{R}}\left(\tilde{\nu}\left(\alpha^{\vee}\right), \eta_{0} \cdot \alpha^{\vee}, \psi_{\mathbf{R}}\right)$ which in turn equals $\gamma\left(\nu\left(\epsilon^{\vee}\right), \tau, \psi_{\mathbf{R}}\right)$.

Proof. Since $\alpha \mid \mathfrak{a}$ is real, $2 \alpha$ cannot be a $a_{0}$-root and therefore by part (a) of Lemma 1.4 the corresponding local coefficient is equal to $\gamma_{\mathbf{R}}\left(\tilde{\nu}\left(\alpha^{\vee}\right), \eta_{0} \cdot \alpha^{\vee}, \psi_{\mathbf{R}}\right)$. Moreover as an $a$-root $2\left\langle\rho_{\alpha}, \alpha\right\rangle /|\alpha|^{2}$ is odd (the number $C$ of pairs of odd (d1) 
roots, restricting to $\alpha / 2 \mid \mathfrak{a}$, is even). Since $\gamma_{\alpha}$ is central, the equality

$$
\gamma_{\mathbf{R}}\left(\tilde{\nu}\left(\alpha^{\vee}\right), \eta_{0} \cdot \alpha^{\vee}, \psi_{\mathbf{R}}\right)=\gamma\left(\nu\left(\epsilon^{\vee}\right), \tau, \psi_{\mathbf{R}}\right)
$$

is now a consequence of Lemma 7.3 of [15], together with Lemma 2.3 of [2] and the equality of infinitesimal characters.

LEMMA 3.12. Let $\alpha$ be a reduced $a_{0}$-root such that $2 \alpha$ is also an $a_{0}$-root. Then $\alpha^{\prime}=2 \alpha$ restricts to a real a-root. Choose a real $\left(\mathfrak{a}_{0} \oplus \mathfrak{m}_{0}\right)_{\mathbf{C}^{-}}$-root $\epsilon^{\prime}$ such that $\epsilon^{\prime} \mid a_{0}=\alpha^{\prime}$. Let $\epsilon=c^{-1}\left(\epsilon^{\prime}\right)$, and denote by $\tau$ the one dimensional restriction of $\rho \cdot \varphi$ to the root space generated by $X_{\epsilon^{\vee}}$. Then in the notation of Lemma 3.2

$$
\gamma_{\mathbf{R}}\left(\tilde{\nu}\left(\alpha^{\vee}+\beta^{\vee}\right),\left(\eta_{0} \cdot\left(\alpha^{\vee}+\beta^{\vee}\right)\right)^{\sim}, \psi_{\mathbf{R}}\right)=\gamma\left(\nu\left(\epsilon^{\vee}\right), \tau, \psi_{\mathbf{R}}\right) .
$$

Proof. Let $\delta=\alpha \mid$ a and $\delta^{\prime}=\alpha^{\prime} \mid$ a. Then by Lemma 7.6 of [15], $\delta^{\prime}$ is real. Let $C$ be the number of root pairs of type (d1) restricting to $\delta$. Since $\alpha$ is an $\mathfrak{a}_{0}$-root, $C=0$. Then by relation (7.25) of [15]

$$
\frac{2\left\langle\rho_{\delta}, \delta^{\prime}\right\rangle}{\left|\delta^{\prime}\right|^{2}} \equiv \frac{2\left\langle\rho_{\alpha}, \alpha^{\prime}\right\rangle}{\left|\alpha^{\prime}\right|^{2}}(\bmod 2)
$$

with obvious meanings for $\rho_{\delta}$ and $\rho_{\alpha}$. Moreover $\gamma_{\alpha^{\prime}}$ is central and therefore the lemma is a consequence of Lemma 7.3 of [15], Lemma 2.3 of [2], and the definition of $\left(\eta_{0} \cdot\left(\alpha^{\vee}+\beta^{\vee}\right)\right)^{\sim}$ (cf. relation (3.14)).

Proof of Theorem 3.1. When $\sigma$ is in the discrete series and $G$ does not have a factor of type $G_{2}$, the theorem is a consequence of relation (3.4), Proposition 3.2.1 of [27], together with Lemmas 3.4, 3.6, 3.9, 3.10, 3.11, and 3.12.

When $G$ has a factor of type $G_{2}$, the discussion in page 437 of [15] shows that Lemmas 3.6 and 3.11 of the present paper, when applied to appropriate roots will prove the theorem. We leave the detail to the reader.

Next assume that $\sigma$ is tempered and $\chi \mid U \cap M_{\theta}^{0}$-generic, $M_{\theta}^{0}=M$. Choose $\theta^{\prime} \subset \theta$, a parabolic subgroup $M \cap P_{\theta^{\prime}}=\left(M \cap A_{\theta^{\prime}}\right) M_{\theta^{\prime}}^{0} \cdot\left(M \cap N_{\theta^{\prime}}\right) \subset M$ a discrete series representation $\sigma^{\prime}$ of $M_{\theta^{\prime}}^{0}$ and an imaginary $\nu^{\prime} \in i a_{\theta^{\prime}}^{*}$ such that $\sigma \hookrightarrow I_{M_{\theta}^{0}}\left(\nu^{\prime}, \sigma^{\prime}, \theta^{\prime}\right)$, the representation induced from $P_{\theta^{\prime}} \cap M_{\theta}^{0}$ to $M_{\theta}^{0}$. Extend $\nu$ to $\tilde{\nu}$ on $\left(\mathfrak{a}_{\theta^{\prime}}\right)_{\mathbf{C}}^{*}$ in the obvious manner. Then

$$
C_{\chi}(\nu, \sigma, \theta, w)=C_{\chi}\left(\tilde{\nu}+\nu^{\prime}, \sigma^{\prime}, \theta^{\prime}, w\right)
$$

and Theorem 3.1 is immediate since the factors attached to $\sigma$ and $\sigma^{\prime} \otimes e^{\nu^{\prime}}$ are the same [21].

Finally assume that $\sigma$ is any irreducible admissible $\chi$-generic Banach representation of $M_{\theta}^{0}$. Choose $\theta^{\prime} \subset \theta$, a tempered representation $\sigma^{\prime}$ of $M_{\theta^{\prime}}^{0}$, and $\nu^{\prime} \in \mathfrak{a}_{\theta^{\prime}}^{*}$ such that $\sigma=I_{M}\left(\nu^{\prime}, \sigma^{\prime}, \theta^{\prime}\right)$ (Theorem 6.2 of [33]). Then (3.27) again holds and for the same reason the theorem is again immediate. This completes the proof of Theorem 3.1. 
To conclude this section we would like to give an analogue of Theorem 3.1 when $\mathbf{G}$ is a complex group and $W_{\mathbf{R}}$ is replaced by $W_{\mathbf{C}}$. The representation $\rho \cdot \varphi$ is now one of $W_{\mathrm{C}}$. Define $r_{i}$ and $a_{i}, i=1, \ldots, n$ as before. We then have:

Theorem 3.13. Suppose $\mathbf{G}$ is a complex group and for any $\varphi: W_{\mathbf{C}} \rightarrow^{L} M$ consider $r_{i}, i=1, \ldots, n$, as representations of $W_{\mathrm{C}}$. Let $\sigma$ be an irreducible admissible $\chi$-generic Banach (in particular unitary $\chi$-generic) representation of $M$. Then

$$
C_{\chi}\left(-2 s \rho_{\theta}, \sigma, \theta, w\right)=\prod_{i=1}^{n} \epsilon\left(a_{i} s, r_{i}, \psi_{\mathrm{C}}\right) \frac{L\left(1-a_{i} s, \tilde{r}_{i}\right)}{L\left(a_{i} s, r_{i}\right)}
$$

4. Identities for intertwining operators. To conclude the paper, we shall prove certain identities which are of importance in deeper analysis of the trace formula (cf. Introduction of [29], where we have proved a similar result for $\mathrm{SL}(r)$ over a $p$-adic field, also see [1]).

As before $G$ is the group of real points of a quasi-split algebraic group over $\mathbf{R}$ and for every $\theta \subset \Delta$, we let $M_{\theta}^{0} A_{\theta} N_{\theta}$ be the Langlands decomposition of $P_{\theta}$. Fix $\chi$ as in Theorem 3.1 and let $(\sigma, H(\sigma))$ be an irreducible tempered $\chi$-generic representation of $M_{\theta}^{0}$. Every tempered $L$-packet contains a unique such representation (cf. [33]). Set

$$
W(\sigma)=\left\{\tilde{w} \in W\left(A_{0}\right) \mid \tilde{w} \sigma \cong \sigma\right\} .
$$

For every $\tilde{w} \in W(\sigma)$, choose a representative $w \in G$ as in Theorem 3.1, and given $\nu \in\left(\mathfrak{a}_{\theta}\right)_{\mathrm{C}}^{*}$ define $A(\nu, \sigma, w)$ by relation (3.2).

Next, for the complex representation $\rho \cdot \varphi$ of $W_{\mathbf{R}}$, let $L(s, \rho \cdot \varphi)$ and $\epsilon\left(s, \rho \cdot \varphi, \psi_{\mathbf{R}}\right)$ be the corresponding Artin $L$-function and root number, respectively. Set (cf. [19])

$$
r(\sigma, w)=\epsilon\left(0, \rho \cdot \varphi, \psi_{\mathbf{R}}\right) L(1, \rho \cdot \varphi) / L(0, \rho \cdot \varphi)
$$

and

$$
R(\nu, \sigma, w)=r(\sigma, w) A(\nu, \sigma, w)
$$

Fix an intertwining operator $\Phi: w \sigma \cong \sigma$ (both acting on $H(\sigma)$ ), and let $\tilde{\Phi}: V(0, w \sigma, \theta) \cong V(0, \sigma, \theta)$ be its lift, i.e., $\tilde{\Phi} f(g)=\Phi(f(g))$. Write

$$
I(0, \sigma, \theta)=\bigoplus_{j=1}^{q} \pi_{j}
$$

Then each $\pi_{j}$ appears with multiplicity one and consequently $\tilde{\Phi} \cdot R(0, \sigma, w)$ acts on each $\pi_{j}$ as a scalar $\epsilon_{\sigma, w}\left(\pi_{j}\right)$. Since $w, d n$, and $\psi_{\mathbf{R}}$ have already been fixed, $\epsilon_{\sigma, w}\left(\pi_{j}\right)$ depends only upon $\Phi$. We remark that the choice of $\Phi$ may be absorbed 
by that of $w$; but we prefer to keep them separated. Our goal is (for a canonically fixed $\Phi)$ to compute each $\epsilon_{\sigma, w}\left(\pi_{j}\right)$.

Next choose $\theta^{\prime} \subset \theta$, a discrete series representation $\sigma^{\prime}$ of $M_{\theta^{\prime}}^{0}$ and $\nu^{\prime} \in \mathfrak{a}_{\theta^{\prime}}^{*}$ such that $\sigma \hookrightarrow I_{M_{\theta}^{0}}\left(\nu^{\prime}, \sigma^{\prime}, \theta^{\prime}\right)$. Then $w \sigma \hookrightarrow I_{M_{\theta}^{0}}\left(\tilde{w} \nu^{\prime}, \tilde{w} \sigma^{\prime}, \tilde{w} \theta^{\prime}\right)$. Consequently, we may assume $\theta^{\prime}=\tilde{w} \theta^{\prime}, \sigma^{\prime}=\tilde{w} \sigma^{\prime}$, and $\nu^{\prime}=\tilde{w} \nu^{\prime}$. Moreover

$$
I(0, \sigma, \theta) \hookrightarrow I\left(\nu^{\prime}, \sigma^{\prime}, \theta^{\prime}\right) .
$$

Let $R$ be the $R$-group for $I\left(\nu^{\prime}, \sigma^{\prime}, \theta^{\prime}\right)$. It is a product of groups of type $\mathbf{Z} / 2 \mathbf{Z}$ which determines the reducibility of $I\left(\nu^{\prime}, \sigma^{\prime}, \theta^{\prime}\right)$ (Theorem 13.4 of [13]). In fact its dual $\hat{R}$ has a simply transitive action on the components of $I\left(\nu^{\prime}, \sigma^{\prime}, \theta^{\prime}\right)$. Let $\pi \hookrightarrow I(0, \sigma, \theta) \hookrightarrow I\left(\nu^{\prime}, \sigma^{\prime}, \theta^{\prime}\right)$ be the unique $\chi$-generic subrepresentation of $I\left(\nu^{\prime}, \sigma^{\prime}\right.$, $\left.\theta^{\prime}\right)$. Choose $1=r_{1}, r_{2}, \ldots, r_{q} \in \hat{R}$ such that $\pi_{j}=r_{j} \cdot \pi, j=1, \ldots, q, \pi_{1}=\pi$. If $\tilde{w} \in W(\sigma)$ is so that $\tilde{\Phi} \cdot R(0, \sigma, w)$ is not a scalar (which happens if $I(0, \sigma, \theta)$ is reducible, cf. Lemma 7.9 and Theorem 13.4 of [13]), then $\tilde{w} \in W\left(\sigma^{\prime} \otimes e^{\nu^{\prime}}\right)$ is in fact in $R$. Observe that

$$
R\left(\nu^{\prime}, \sigma^{\prime}, w\right) \mid V(0, \sigma, \theta)=R(0, \sigma, w) .
$$

Finally, let $\lambda$ be a $\chi$-Whittaker functional for $H(\sigma)_{\infty}$. We fix $\Phi$ such that $\langle\lambda, v\rangle=\langle\lambda, \Phi v\rangle$ for every $v \in H(\sigma)_{\infty}$. Then $\Phi$ is unique and independent of the choice of $\lambda$. Now, for every $j, j=1, \ldots, q$, let $\chi_{j}$ be the tempered character of $\pi_{j}$. In what follows, for every $f \in C_{c}^{\infty}(G)$, we let

$$
I(\sigma, \theta) f(g)=\int_{G} f(g) I(0, \sigma, \theta)(g) d g .
$$

TheOREM 4.1. Fix $\tilde{w} \in W(\sigma)$ and choose $\Phi, \psi_{\mathbf{R}}, d n$, and $w$ as above. Let $m$ and $p$ be the number of rank one groups $G_{\alpha}$, $\alpha$ reduced, $\alpha \in \Sigma_{\theta}^{+}, \tilde{w}(\alpha) \in \Sigma^{-}$, whose simply connected coverings are isomorphic to $\mathrm{SU}(2,1)$ and $\mathrm{SL}(2, \mathrm{C})$, respectively.

(a) Suppose $\tilde{\Phi} \cdot R(0, \sigma, w)$ is a scalar. Then

$$
\operatorname{trace}(\tilde{\Phi} \cdot R(0, \sigma, w) I(\sigma, \theta)(f))=\lambda\left(\mathbf{C} / \mathbf{R}, \psi_{\mathbf{R}}\right)^{2 m+p} \sum_{j=1}^{q} \chi_{j}(f) .
$$

(b) Suppose $\tilde{\Phi} \cdot R(0, \sigma, w)$ is not a scalar. Regard $\tilde{w}$ as an element of $R$. Then

$$
\operatorname{trace}(\tilde{\Phi} \cdot R(0, \sigma, w) I(\sigma, \theta)(f))=\lambda\left(\mathbf{C} / \mathbf{R}, \psi_{\mathbf{R}}\right)^{2 m+p} \sum_{j=1}^{q} r_{j}(\tilde{w}) \chi_{j}(f) .
$$

Proof. In view of Theorem 3.1, the theorem is a consequence of a result of P. Delorme [3] and Lemma 4.2 below. In fact, by part (iv) of Theorem 1 of [3]

$$
\epsilon_{\sigma, w}\left(\pi_{j}\right) / \epsilon_{\sigma, w}(\pi)=r_{j}(\tilde{w}),
$$

and the proof reduces to calculating $\epsilon_{\sigma, w}(\pi)$. But by Theorem 3.1 (setting $s=0$ in (3.1.1)) this follows immediately from the following lemma. 
Lemma 4.2. Suppose $\sigma \cong \tilde{w} \sigma$. Then $L(1, \rho \cdot \varphi)$ and $L(1, \tilde{\rho} \cdot \varphi)$ are both finite and equal.

Proof. By definition of Artin $L$-functions we may assume $\sigma$ is in the discrete series. Let $\epsilon$ be an $\left(a_{\theta}+\mathfrak{b}\right)_{C}$-root. Then by (3.18)

$$
\epsilon^{\vee}(\varphi(z))=(z \bar{z})^{\left(\left\langle\mu, \epsilon^{\vee}\right\rangle+\left\langle\bar{\sigma} \mu, \epsilon^{\vee}\right\rangle\right) / 2}(z / \bar{z})^{\left(\left\langle\mu, \epsilon^{\vee}\right\rangle-\left\langle\bar{\sigma} \mu, \epsilon^{\vee}\right\rangle\right) / 2},
$$

where $\left\langle\mu, \epsilon^{\vee}\right\rangle-\left\langle\bar{\sigma} \mu, \epsilon^{\vee}\right\rangle$ is an integer [21]. Now, by Lemma 5.3 .14 of [32], $\tilde{w}$ may be realized as an element $l$ in the centralizer of $\varphi\left(W_{\mathbf{R}}\right)$, as well as in the normalizer of ${ }^{L}\left(B A_{\theta}\right)^{0}$, both in ${ }^{L} G^{0}$. Then for $z \in \mathbf{C}^{*} \subset W_{\mathbf{R}}, \varphi(z) l$ sends $X_{\epsilon^{\vee}}$ to $\left(l\left(\epsilon^{\vee}\right)\right)(\varphi(z)) X_{l\left(\epsilon^{\vee}\right)}$ which must be equal to $\epsilon^{\vee}(\varphi(z)) X_{l\left(\epsilon^{\vee}\right)}$. Consequently

$$
\epsilon^{\vee}(\varphi(z))=l\left(\epsilon^{\vee}\right)(\varphi(z)) .
$$

Now, observe that $\tilde{\rho} \cdot \varphi$ may be realized as the adjoint action of $\varphi\left(W_{\mathbf{R}}\right)$ on $\left\{X_{l\left(\epsilon^{\vee}\right)}, \epsilon^{\vee} \in{ }^{L} \mathfrak{n}_{\tilde{w}}\right\}$. Set

$$
t=\left\langle\mu, \epsilon^{\vee}\right\rangle+\left\langle\bar{\sigma} \mu, \epsilon^{\vee}\right\rangle
$$

and

$$
n=\left\langle\mu, \epsilon^{\vee}\right\rangle-\left\langle\bar{\sigma} \mu, \epsilon^{\vee}\right\rangle \text {. }
$$

Denote by $l(t)$ and $l(n)$, the values of (4.2.2) and (4.2.3), when $\epsilon^{\vee}$ is replaced by $l\left(\epsilon^{\vee}\right)$, respectively. Then by $(4.2 .1), t=l(t)$ and $n=l(n)$.

Suppose first that $\epsilon$ is complex. Then $\rho \cdot \varphi \mid\left\{X_{\epsilon^{\vee}}, X_{\bar{\epsilon}^{\vee}}\right\}$ and $\tilde{\rho} \cdot \varphi \mid\left\{X_{l\left(\epsilon^{\vee}\right)}\right.$, $\left.X_{l\left(\bar{\epsilon}^{\vee}\right)}\right\}$ are both the irreducible representation induced by $|z|_{\mathbf{C}}^{t / 2}(z / \bar{z})^{n / 2}$ from $\mathbf{C}^{*}$ to $W_{\mathbf{R}}$, and therefore

$$
L\left(s, \rho \cdot \varphi \mid\left\{X_{\epsilon^{\vee}}, X_{\bar{\epsilon}^{\vee}}\right\}\right)=L\left(s, \tilde{\rho} \cdot \varphi \mid\left\{X_{l\left(\epsilon^{\vee}\right)}, X_{l\left(\bar{\epsilon}^{\vee}\right)}\right\}\right) .
$$

Next, assume $\epsilon$ is real. Then $\varphi(1 \times \sigma) l$ sends $X_{\epsilon} \vee$ to $c_{l\left(\epsilon^{\vee}\right)} X_{l\left(\epsilon^{\vee}\right)}$ which must be equal to $c_{\epsilon^{\vee}} X_{l\left(\epsilon^{\vee}\right)}$, proving $c_{\epsilon^{\vee}}=c_{l\left(\epsilon^{\vee}\right)}, c_{\epsilon^{\vee}}= \pm 1$. Moreover, using (4.2.1), one concludes that $t=l(t)=2\left\langle\mu, \epsilon^{\vee}\right\rangle$. Writing $x=(x /|x|) z \bar{z}, z \in \mathbf{C}^{*}$, we see, using class field theory, that $\rho \cdot \varphi \mid\left\{X_{\epsilon^{\vee}}\right\}$ is in fact the character $\tau$ of $\mathbf{R}^{*}$, given by

$$
\left.\tau(x)=(x /|x|)^{1 / 2\left(1-c_{\varepsilon} \vee\right.}\right)(z \bar{z})^{\left\langle\mu, \epsilon^{\vee}\right\rangle} .
$$

It is then clearly equivalent to $\tilde{\rho} \cdot \varphi \mid\left\{X_{l\left(\epsilon^{\vee}\right)}\right\}$. Observe that we have in fact proved the equivalence of $\rho \cdot \varphi$ and $\tilde{\rho} \cdot \varphi$ under the assumption $\sigma \cong \tilde{w} \sigma$.

The finiteness of $L(1, \rho \cdot \varphi)$ is now a consequence of the fact that, for every $\epsilon$, corresponding $t$ is imaginary.

\section{REFERENCES}

1. J. Arthur, "On some problems suggested by the Trace Formula," Lie Group Representations II, (Lecture Notes in Math. 1041, Springer-Verlag, 1983), 1-49. 
2. - On the invariant distributions associated to weighted orbital integrals, preprint.

3. P. Delorme, Homomorphismes de Harish-Chandra lies aux K-types minimaux des series principales generalisees des groupes de Lie reductifs connexes, Ann. Sci. École Norm. Sup. 17 (1984), 117-156.

4. R. Goodman and N. R. Wallach, Whittaker vectors and conical vectors, J. Functional Anal. 39 (1980), 199-279.

5. M. HaShizUme, Whittaker models for real reductive groups, Japan J. Math. 5 (1979), 349-401.

6. H. JACQUET, Fonctions de Whittaker associeés aux groups de Chevalley, Bull. Soc. Math. France 95 (1967), 243-309.

7. H. JACQUeT AND J. A. Shalika, On Euler products and the classification of automorphic representations I \& II, Amer. J. Math. 103 (1981), 449-558 \& 777-815.

8. H. Jacquet, I. I. Piatetski-Shapiro and J. A. Shalika, Rankin-Selberg convolutions, Amer. J. Math. 105 (1983), 367-464.

9. D. Keys, Principal series representations of special unitary groups over local fields, Comp. Math. 51 (1984), 115-130.

10. A. W. KnaPP, Weyl group of a cuspidal parabolic, Ann. Sci. Ecole Norm. Sup. 8 (1975), 275-294.

11. Commutativity of intertwining operators for semisimple groups, Comp. Math. 46 (1982), 33-84.

12. AND E. M. STEIN, Intertwining operators for semisimple groups, Ann. of Math. 93 (1971), 489-578.

13. _ Intertwining operators for semisimple groups II, Invent. Math. 60 (1980), 9-84.

14. A. W. KNAPP AND N. R. WALlaCh, Szegö kernels associated with discrete series, Invent. Math. 34 (1976), 163-200.

15. A. W. KNAPP AND G. J. ZUCKERMAN, Classification of irreducible tempered representations of semisimple groups, Ann. of Math. 116 (1982), 389-455.

16. B. Kostant, On Whittaker vectors and representation theory, Invent. Math. 48 (1978), 101-184.

17. J. P. Labesse AND R. P. Langlands, L-indistinguishability for SL(2), Can. J. Math. 31 (1979), $726-785$.

18. R. P. Langlands, On Artin's L-functions, Rice University Studies 56 (1970), 23-28.

19. - On the Functional Equation Satisfied by Eisenstein Series, (Lecture Notes in Math. 544, Springer-Verlag, 1976).

20. —— Euler Products, Yale Univ. Press, New Haven, 1971.

21. Advanced Study, Mimeographed notes, 1973.

22. C. J. Moreno AND F. Shahid, The L-function $L_{3}\left(s, \pi_{\Delta}\right)$ is entire, Invent. Math. 79 (1985), 247-251.

23. N. S. PoulSEN, On $C^{\infty}$-vectors and intertwining bilinear forms for representations of Lie groups, J. Functional Anal. 9 (1972), 87-120.

24. G. Schiffmann, Intégrales d'enterlacement et fonctions de Whittaker, Bull. Soc. Math. France 99 (1971), 3-72.

25. F. SHAHIDI, Functional equation satisfied by certain L-functions, Comp. Math. 37 (1978), 171-208.

26. ——, Whittaker models for real groups, Duke Math. J. 47 (1980), 99-125.

27. _- On certain L-functions, Amer. J. Math. 103 (1981), 297-355.

28. - Fourier transforms of intertwining operators and Plancherel measures for GL( $n)$, Amer. J. Math. 106 (1984), 67-111.

29. _- Some results on L-indistinguishability for SL(r), Can. J. Math. 35 (1983), 1075-1109.

30. - Artin L-functions and normalization of intertwining operators, Seminar on the Analytical Aspects of the Trace Formula II, Institute for Advanced Study, 1983-84.

31. J. A. ShalikA, The multiplicity one theorem for $\mathrm{GL}_{n}$, Ann. of Math. 100 (1974), 171-193.

32. D. ShelstaD, L-indistinguishability for real groups, Math. Ann. 259 (1982), 385-430.

33. D. A. VogAN, Gelfand-Kirillov dimension for Harish-Chandra modules, Invent. Math. 48 (1978), 75-98. 
34. N. R. Wallach, "Asymptotic expansions of generalized matrix entries of representations of real reductive groups," Lie Group Representations I, (Lecture Notes in Math. 1024, Springer-Verlag, 1983), 287-369.

35. E. T. Whittaker and G. N. Watson, A Course in Modern Analysis, Cambridge Univ. Press, London, 1963.

36. N. R. WALLACH, Lie algebra cohomology and holomorphic continuation of generalized Jacquet integrals, preprint.

Department of Mathematics, Purdue University, West Lafayette, Indiana 47907 